\title{
Effect of surface roughness on the ultrashort pulsed laser ablation fluence threshold of zinc and steel
}

\author{
H. Mustafa, ${ }^{a, *}$, M. Mezera ${ }^{a}$, D.T.A. Matthews ${ }^{a, b, c}$, G.R.B.E. Römer ${ }^{a}$ \\ ${ }^{a}$ Chair of Laser Processing, Department of Mechanics of Solids, Surfaces 83 Systems (MS ${ }^{3}$ ), Faculty of \\ Engineering Technology, University of Twente, Enschede, the Netherlands \\ ${ }^{b}$ Chair of Skin Tribology, Department of Mechanics of Solids, Surfaces \& Systems (MS ${ }^{3}$ ), Faculty of \\ Engineering Technology, University of Twente, Enschede, the Netherlands \\ ${ }^{c}$ Research $\& 3$ Development, Tata Steel, PO Box 10000, 1970 CA IJmuiden, the Netherlands
}

\begin{abstract}
The single and multiple pulse laser ablation threshold of zinc and steel at picosecond laser pulse duration is studied as a function of initial surface roughness at laser wavelengths of 515 and $1030 \mathrm{~nm}$. The initial surface topographies and the resulting crater morphologies are analyzed using confocal laser scanning microscopy (CLSM) and scanning electron microscopy (SEM). Reflectivity measurements of the initial surfaces show increased absorptivity with increasing surface roughness. It was found that the single pulse ablation threshold increases with increasing effective surface area; the latter resulting from surface roughness. Rougher surfaces tend to have a higher degree of incubation as well. From the experimental and simulation results, it appears that the absorbed energy contributes more to residual heat than to material ablation when effective surface area increases.
\end{abstract}

Keywords: ultrashort pulsed laser, ablation threshold, surface roughness, polycrystalline zinc, titanium stabilized ultra low carbon steel, galvanized steel

\section{Introduction}

Laser ablation is a subtractive micromachining technique, which can be employed to improve the surface functionality of a product by applying a laser-induced texture to the surface [1]. It is a flexible and precise manufacturing process compared to other techniques, such as electric discharge texturing, chemical etching, shot blasting and electron beam texturing [2]. The absorption of laser light and subsequent heating of the material being processed depends not only on the optical properties of the material, but also on its initial surface condition [3]. This means features such as roughness, oxidation, impurities, defects etc., of the targeted material play a major role in the efficiency of material removal and ultimately the resulting quality of the machined surface. A higher surface roughness typically results

\footnotetext{
*Corresponding author at: P.O. Box 217, 7500 AE, Enschede, the Netherlands

Email address: h.mustafa@utwente.nl (H. Mustafa)
} 
in higher absorption of optical (laser) energy through scattering related phenomena from surface irregularities and asperities, such as multiple reflection, shadowing, and back and side scattering [4 6]. Although for continuous wave $(\mathrm{cw})$ laser processing, e.g. during laser welding, no dependency between the surface roughness and process efficiency is observed for wavelengths in the far IR regime $(\geq 10 \mu \mathrm{m})$ [7, 8], weld penetration depth is found to increase with increasing surface roughness for NIR wavelengths $(\leq 1.5 \mu \mathrm{m})$ [8]. A higher degree of optical heating is also reported for increasing roughness [5]. Moreover, absorption is a function of temperature of the material, and generally increases with increasing temperature until structural transformation of the rough surface takes place [9]. In the case of ultra short laser pulses, with pulse durations in the picosecond regime, or shorter, the absorption of laser energy (photons) and modification of the material, such as ablation, take place at different time scales. That is, absorption of photons typically takes place on the femto- to picosecond time scale, whereas modification of material takes place on the hundreds of picoseconds to nanosecond timescale or longer [10]. From the perspective of light absorption, surface roughness can be interpreted as the deviations in local angle of incidence (AOI, $\left.\theta_{i}[\mathrm{deg}]\right)$ from ideal flat surface. This geometric change in local AOI $\left(\theta_{R}\right)$ decreases the amount of absorbed fluence proportional to $\cos \left(\theta_{i} \pm \theta_{R}\right)$, which in turn, results in nonuniform temperature distributions over local surfaces [11. For laser intensities $<10^{14} \mathrm{~W} / \mathrm{cm}^{2}$, collisional absorption mechanism, such as inverse Bremsstrahlung (IB), dominates for smooth surfaces; whereas, with increasing surface roughness, the absorption is affected by the micro and nano scale surface irregularities through plasmonic absorption [12]. From atomistic modeling, surface roughness is found to lower effective thermal conductivity, due to increase in surface area to volume ratio [13]. Variations in the temperature distribution result in a decrease in ablation efficiency with increasing surface roughness [14], as well as false signal increase in laser-induced breakdown spectroscopy (LIBS) [15]. Until a well-defined, stable structure, like an ablated crater, is formed, the ablation rate [11] as well as ablation plume deflection [11, 16, 17] varies with increasing number of pulses. For optical surfaces, laser induced damage threshold is found to decrease with increasing atomic level $(\AA)$ roughness [18, 19]. In other words, surface roughness becomes a critical factor for laser processing materials with a low number of pulses. However, for engineering surfaces, the influence of surface roughness (at the $\mu \mathrm{m}$ scale) on the laser ablation threshold, as yet, has not been well-quantified.

Therefore, in this work, the effect of surface roughness on the fluence threshold (that is, the fluence level above which ablation occurs) in metallic materials in the ultrashort pulse regime is studied. Since there is a substantial difference in laser processing results between metals in pure and coated form, three different metallic materials namely, bulk metal (zinc), metallic coating (galvanized steel) and metallic alloy (forming steel), are chosen to identify the effect of surface roughness on fluence ablation threshold. Ablation of bulk zinc, galvanized steel and forming steel is performed with single, as well as multiple, picosecond laser pulses at wavelengths of $1030 \mathrm{~nm}$ and $515 \mathrm{~nm}$ and at different preliminary surface roughness $\left(R_{a}\right)$ values ranging from $0.02 \mu \mathrm{m}$ to $1.3 \mu \mathrm{m}$. Absorptivity of different unprocessed surfaces is measured prior to laser processing. From the laser processed surfaces, the ablation thresh- 
olds and incubation coefficients are calculated. In addition, one dimensional ray tracing and heat accumulation models are presented in relation to different surface roughnesses.

\section{Experimental Setup}

\subsection{Laser setup}

The laser ablation experiments were performed under atmospheric conditions in a cleanroom environment, using a diode pumped thin disc Yb:YAG pulsed laser source (TruMicro 5050 of Trumpf GmbH, Germany). This source emits 6.7 ps laser pulses of linearly polarized light and shows a nearly Gaussian power density profile $\left(M^{2}<1.3\right)$. In this work, $p$-polarized light at both the central $(1030 \mathrm{~nm})$ and the second harmonic $(515 \mathrm{~nm})$ wavelengths was used. A galvo-scanner (IntelliScan14 of ScanLab GmbH, Germany), equipped with a telecentric flatfield F-theta-Ronar lens (Linos GmbH, Germany) was used to scan the focused laser beam over the surface of the sample. The sample was placed in the focal plane. The focal spot radius was measured from the fluence profile using a charge-coupled device (CCD) sensor-based, beam diagnostic system (MicroSpot Monitor of Primes GmbH, Germany). The setup related parameters are listed in Table 1.

Table 1: Laser setup parameters

\begin{tabular}{|c|c|c|}
\hline \multirow{2}{*}{ Parameters } & \multicolumn{2}{|c|}{ Laser wavelength $[\mathrm{nm}]$} \\
\hline & 1030 & 515 \\
\hline Focal length $[\mathrm{mm}]$ & 80 & 100 \\
\hline Focal spot radius $[\mu \mathrm{m}]$ & $14.6 \pm 1.6$ & $12.0 \pm 0.5$ \\
\hline Ellipticity@ focus & 0.89 & 0.81 \\
\hline Max. pulse energy $[\mu \mathrm{J}]$ & 135 & 62 \\
\hline Min. pulse energy $[\mu \mathrm{J}]$ & 3 & 1 \\
\hline
\end{tabular}

The beam impinges perpendicular to the sample surface. The focus position was fixed for all the experiments and coincided with the original surface. An exhaust system was used to extract debris from the laser material interaction zone during processing. The laser energy supplied to the surface was varied by using a combination of a half-wave plate and a polarizing beam splitter. A pyroelectric detector (PM30 with FieldMax II of Coherent, USA) was used to measure the average laser power incident on the sample with an error less than $8 \%$. The energy of the individual pulses was determined by dividing the measured average laser power by the pulse frequency applied. The power instability of the laser source is less than $2 \%$. At a repetition rate of $8 \mathrm{kHz}$ and a beam scanning velocity of $1 \mathrm{~m} / \mathrm{s}$, time between consecutive pulses on the same location equals at least $3.9 \mathrm{~ms}$. The geometrical pulse-to-pulse distance was at least $125 \mathrm{\mu m}$ and the number of pulses varied from $N=1$ to 50. A total of 21 craters were created per laser setting to ensure statistically sound values in measured quantities. 


\subsection{Material}

Three different materials were used in this work namely - $(i)$ bulk polycrystalline zinc (Zn), (ii) galvanized steel (GI) and ( $i i i)$ forming steel (FS). The galvanized steel consists of a zinc coating on forming steel, having an average thickness of $8 \pm 2 \mu \mathrm{m}$, deposited by Hot Dip Galvanizing (HDG) process according to European standard EN10346:2015. The forming steel, in this work, is Titanium Stabilized Ultra Low Carbon (TiSULC) steel. The chemical composition of these materials are listed in Table 2. To achieve different surface roughnesses, these samples were subjected to different surface treatments as listed in Table 3. After surface treatments, Zn, GI and FS samples were cleaned with ethanol ( $>99 \%)$, ammonia $(<5 \%)$ and acetic acid $(<20 \%)$ respectively and dried with a stream of cold air. Material specific thermal properties of these materials are listed in Table 4.

Table 2: Chemical composition of materials ( $w t \%)$

\begin{tabular}{llccccccc}
\hline Sample & Material & $\mathrm{C}$ & $\mathrm{Mn}$ & $\mathrm{P}$ & $\mathrm{S}$ & $\mathrm{Fe}$ & $\mathrm{Zn}$ & $\mathrm{Al}$ \\
\hline FS1-FS2 & Forming steel & $<0.12$ & $<0.6$ & $<0.1$ & $<0.045$ & Balance & - & - \\
Zn1-Zn2 & Zinc & - & - & - & - & - & Balance & $<0.5$ \\
GI1-GI5 & Galvanized steel & - & - & - & - & - & 99.8 & 0.2 \\
\hline
\end{tabular}

Table 3: Surface treatments and resulting surface roughnesses

\begin{tabular}{|c|c|c|c|c|c|c|}
\hline Material & Zinc & Forn & ing steel & \multicolumn{3}{|c|}{ Galvanized Steel } \\
\hline Surface & Casted & \multicolumn{2}{|c|}{ Cold-rolled } & \multicolumn{3}{|c|}{$\longleftrightarrow$ Hot dipped $\longrightarrow$} \\
\hline Sample & Zn1 Zn2 & FS1 & FS2 & GI1 & GI3 & GI4 \\
\hline Treatment & $\leftarrow$ Polishin & $g \rightarrow$ & Pickling & Polishing & As received & Sand-blasting \\
\hline$R_{a}[\mu \mathrm{m}]$ & $0.03 \quad 0.02$ & 0.15 & 1.26 & $0.09 \quad 0.21$ & $<0.5$ & 0.62 \\
\hline
\end{tabular}

Table 4: Thermal properties of zinc 20] and forming steel 21], namely density $(\rho)$, specific heat $\left(C_{p}\right)$, thermal conductivity $(K)$, melting temperature $\left(T_{m}\right)$, vaporization temperature $\left(T_{v}\right)$, latent heat of melting $\left(L_{m}\right)$ and vaporization $\left(L_{v}\right)$.

\begin{tabular}{cccccccc}
\hline Material & $\rho\left[\mathrm{kg} / \mathrm{m}^{3}\right]$ & $C_{p}[\mathrm{~kJ} /(\mathrm{kg} \cdot \mathrm{K})]$ & $K[\mathrm{~W} /(\mathrm{m} \cdot \mathrm{K})]$ & $T_{m}[\mathrm{~K}]$ & $T_{v}[\mathrm{~K}]$ & $L_{m}[\mathrm{~kJ} / \mathrm{kg}]$ & $L_{v}[\mathrm{~kJ} / \mathrm{kg}]$ \\
\hline Zinc & 7140 & 0.382 & 113 & 692.68 & 1180 & 100.9 & 1782 \\
Forming steel & 7840 & 0.506 & 62.8 & 1803 & - & 275 & - \\
\hline
\end{tabular}

\subsection{Analysis tools}

In order to obtain the optical constants, i.e. the refractive index $n$ and extinction coefficient $k$, of the polished samples, spectroscopic ellipsometry measurement (M-2000UI ellipsometer from Woollam, USA) was carried out on the untreated surface over a wavelength range from 245 to $1690 \mathrm{~nm}$ at $65^{\circ}, 70^{\circ}, 75^{\circ}$ incident angles. The reflection spectra of the unprocessed samples were measured using an integrating sphere (UPB-150-ART of GigahertzOptik, Germany). Broadband light (300-2500 nm) from a Tungsten-Halogen Light Source 
(AvaLight-HAL-S of Avantes, the Netherlands) is guided by fiber optic, focused by an achromatic doublet lens on the sample and subsequent reflection spectrum is collected by a spectrometer (HR-4000 of Ocean Optics, USA). $\mathrm{Ba}_{2} \mathrm{SO}_{4}$ was used as a reference standard.

The roughness of samples after surface treatments, as well as the laser-induced surface profiles (the latter referred to as 'craters 'here), were measured by means of Confocal Laser Scanning Microscopy (CLSM), (VK-9700 of Keyence Corporation, Japan). The lateral and vertical resolution of CLSM measurements was $276 \mathrm{~nm}$ and $1 \mathrm{~nm}$ respectively. Laser-induced crater morphology was analyzed by means of a field emission Scanning Electron Microscope (SEM), (JSM-7200F of Jeol, USA). In order to determine the periodicity of laser-induced periodic surface structures (LIPSS), SEM micrographs were analyzed with the help of a 2D Fast Fourier Transform (FFT) algorithm including normalization into the laser wavelength using a MATLAB script. This algorithm converts the spatial image information (periodicity of LIPSS) into the frequency domain to allow robust determination of the periodicity of LIPSS. The script allows filtering of noise to increase the accuracy at which the LIPSS periodicity is determined.

\section{Results}

In the following, we discuss the results of the samples prior to, and after laser processing. We start with surface metrology to analyze and quantify surface roughness related parameters before laser processing in Sec 3.1. This lays the foundation for analyzing the effect of different surfaces and their corresponding features (e.g. peaks, valleys, depressions etc.) on subsequent laser ablation. Since laser processing involves optical energy, the reflectivity/absorptivity of the sample surfaces primarily determines the amount of available energy for material removal. Therefore, we measured the optical reflectivity of all 9 samples prior to laser processing in Sec. 3.2. These two subsections, i.e. Sec. 3.2 and 3.2 , describe the surface characteristics before laser processing. After the laser processing, we analyze the ablated crater morphology in Sec. 3.3. Next, we calculated the ablation thresholds and incubation coefficients from the dimensional measurements of the ablated craters in Sec. 3.4. Finally, in Sec. 4, we present our possible explanations for observed phenomena and discuss the results described in this section.

\subsection{Surface topography}

In this section, surface topography of different material surfaces after surface treatments, such as mechanical polishing, abrasive blasting or chemical etching (see Table 3), are discussed. These surfaces act as the reference surface before laser processing. In the majority of the literature dealing with laser processing, surface irregularities are characterized by two amplitude parameters, namely average $\left(R_{a}=1 / l \int_{0}^{l}|z(x)| d x\right)$ and root mean square $\left(\sigma=\sqrt{1 / l \int_{0}^{l}\{z(x)\}^{2} d x}\right)$ roughness, to specify the initial surface roughness [5, 14, 15, 22, 24]. However, the height distributions alone cannot describe the surface completely, and spatial parameters like the autocorrelation length is required to completely characterize a surface [25, 26]. Absorption of laser light is reported to increase with the RMS slope $\sigma / \tau$, where $\sigma$ 

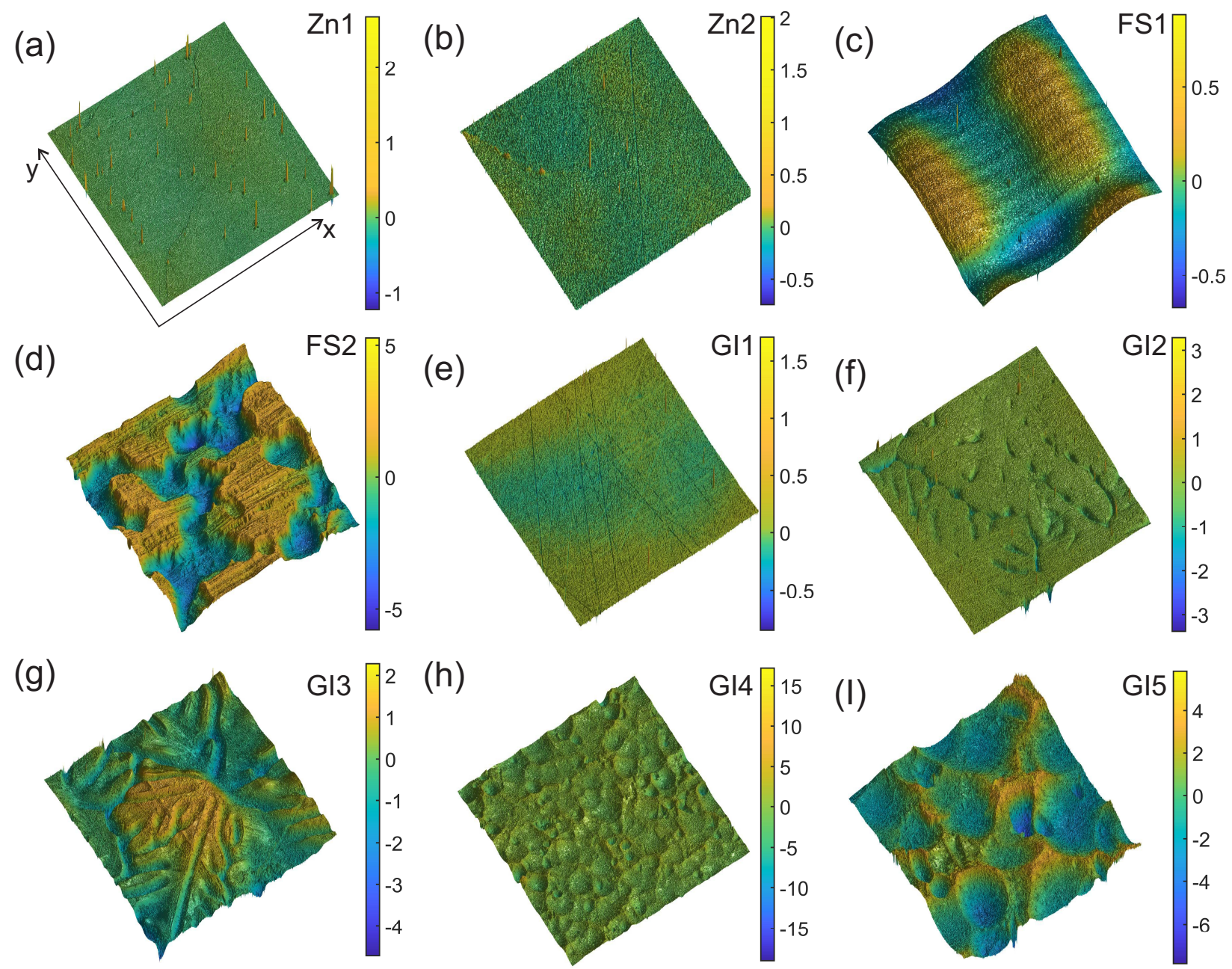

Figure 1: 3D height profiles, as measured from CLSM, of different material surfaces for an area of $200 \times$ $200 \mu^{2}$ after surface treatment. The color scale is in $\mu \mathrm{m}$ and the $x$ and $y$ axis shown in (a) applies to all the graphs.

is RMS roughness and $\tau$ is autocorrelation length [4. In Fig. 1, representative 3D height profiles of all the surface conditions used in this work are shown, see also Table 5. As can be observed, different surfaces have different kinds of surface textures. Presence of micro and nano scale scratches from polishing can be observed in Fig. 1(b) in comparison to Fig. 1(a). Wavy appearance in Fig. 1(c) results from vibrations in the polishing tool. The rolling direction is visible on the pickled forming steel sample in Fig. 11(d). The as received hot dipped galvanized steel surface features dendritic structures, analogous to depressions, over the surface as shown in Fig. 1 (g). Polishing these dendritic structures results in lower surface roughness (see Fig. 1(e), 1(f) and Table 5). Sand blasting of the galvanized surfaces also results in removing dendritic structures and increasing roughness through depressions of different sizes due to a different sandblasting pressure (see Fig. 1(h) and (i)). For all the 
samples, surface related parameters are listed in Table 5. Due to the non-isotropic nature of the surfaces, the autocorrelation length $\tau$ is different along the $x$ and $y$ direction. Compared to a flat surface, any surface roughness does increase the actual surface area $(S A)$ within a projected area $(A)$. The projected area $(\mathrm{A})$ is the two dimensional plane resulting from the field of view of the confocal microscope. In our case, working at a magnification of 50X results in a projected area of $200 \times 200 \mu^{2}$. Since optical heating of the material mainly happens at the surface, the ratio $S A / A$ of the actual surface area over a projected area is also mentioned in Table 5 for an area of $200 \times 200 \mu \mathrm{m}^{2}$. This ratio $S A / A$ is 1 for a perfectly flat surface. The increase in actual surface area compared to projected area is calculated as $\Delta_{S A / A}=(S A / A-1) \times 100 \%$. It can be concluded from Table 5 that, with increasing average roughness $\left(R_{a}\right)$, all other surface parameters increase as well. However, effective surface area, which is the area exposed to laser beam, plays a dominant role for material ablation [19. Moreover, $\Delta_{S A / A}$ is a dimensionless quantity and, therefore, is independent of scale. For this reason, the results of the subsequent sections are compared with respect to $\Delta_{S A / A}$. The amplitude (height) density function (ADF) and inclination angle distribution (IAD)

Table 5: Surface roughness parameters

\begin{tabular}{|c|c|c|c|c|c|c|}
\hline Specimen & Material & \multicolumn{2}{|c|}{$[\mu \mathrm{m}]$} & $\sigma / \tau_{X}$ & $\begin{array}{c}\sigma / \tau_{Y} \\
{[-]}\end{array}$ & $S A / A$ \\
\hline $\mathrm{Zn} 1$ & \multirow{2}{*}{$\mathrm{Zn}$} & $0.03 \pm 0.004$ & $0.03 \pm 0.004$ & $0.0015 \pm 0.0002$ & $0.0021 \pm 0.0003$ & $1.0016 \pm 0.0003$ \\
\hline $\mathrm{Zn} 2$ & & $0.02 \pm 0.006$ & $0.03 \pm 0.007$ & $0.1087 \pm 0.0254$ & $0.1087 \pm 0.0254$ & $1.0061 \pm 0.0028$ \\
\hline GI1 & \multirow{5}{*}{ GI } & $0.09 \pm 0.02$ & $0.12 \pm 0.03$ & $0.0051 \pm 0.0015$ & $0.0071 \pm 0.0021$ & $1.0205 \pm 0.0022$ \\
\hline GI2 & & $0.21 \pm 0.03$ & $0.26 \pm 0.03$ & $0.0264 \pm 0.0031$ & $0.0417 \pm 0.0048$ & $1.047 \pm 0.0121$ \\
\hline GI3 & & $0.22 \pm 0.03$ & $0.28 \pm 0.03$ & $0.0426 \pm 0.0043$ & $0.0465 \pm 0.0047$ & $1.0641 \pm 0.0133$ \\
\hline GI4 & & $0.62 \pm 0.05$ & $0.81 \pm 0.08$ & $0.0697 \pm 0.0069$ & $0.0733 \pm 0.0073$ & $1.1027 \pm 0.0162$ \\
\hline GI5 & & $1.04 \pm 0.05$ & $1.32 \pm 0.07$ & $0.0855 \pm 0.0047$ & $0.0887 \pm 0.0049$ & $1.2784 \pm 0.0196$ \\
\hline FS1 & \multirow{2}{*}{ FS } & $0.15 \pm 0.01$ & $0.19 \pm 0.02$ & $0.0099 \pm 0.001$ & $0.008 \pm 0.0008$ & $1.0009 \pm 0.0002$ \\
\hline FS2 & & $1.26 \pm 0.2$ & $1.49 \pm 0.24$ & $0.0459 \pm 0.0072$ & $0.0444 \pm 0.007$ & $1.0357 \pm 0.0139$ \\
\hline
\end{tabular}

of these surfaces reveals additional information about the surface features for estimating the geometric change in local AOI $\left(\theta_{R}\right)$. For example, ADFs of nearly all the samples exhibit depressions and scratches rather than peaks and spikes, which implies that $\theta_{R}$ will be nonzero (see Supplementary material Fig. S.1 and S.2]. Moreover, in spite of having almost similar $\sigma$, the wider IAD for GI5 indicates that the transitions between local peaks and valleys are more steep for sample FS2 than for sample GI5 (see Supplementary material Fig. S.2). This implies that grazing incidence will be dominant for FS2.

\subsection{Surface reflectivity}

In order to investigate the effect of surface roughness on optical absorption, reflectivity of different surfaces prior to laser processing are measured and modeled. The absorptivity $A$ of a material surface is the reminder after transmission $T$, reflection $R$ and scattering $S$. Since $T=0$ for metals with sufficient thickness $(\geq 100 \mathrm{~nm})$, absorptivity $A$ is given by $A=1-R-S[5]$. In other words, specular and diffuse reflectance ends up as $R$ and $S$. 

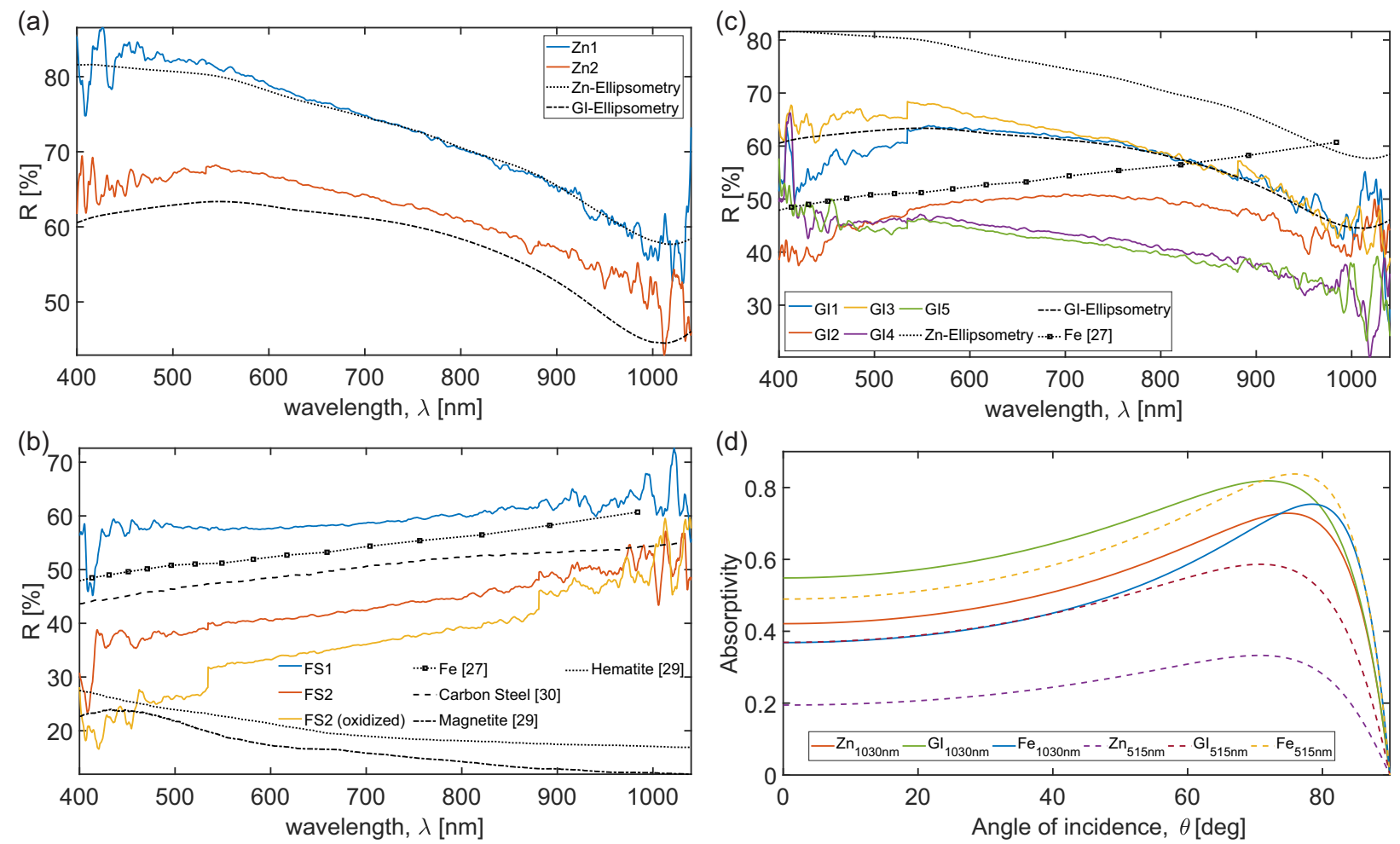

Figure 2: Reflectivity $R$ as a function of wavelength $\lambda$ of different material surfaces measured using the integrating sphere setup, namely (a) zinc (b) forming steel and (c) galvanized steel. (d) Fresnel plot of absorptivity $A$ for the three materials irradiated at 515 and $1030 \mathrm{~nm}$ wavelength as a function of angle of incidence $\theta_{i}$ relative to the surface normal. Data for Fe is taken from [27].

Figure 2(a)-(c) shows the reflectivity of our samples measured using an integrating sphere setup at the 8/d configuration. Optical constants $n$ and $k$ of pure zinc and galvanized steel were measured by ellipsometry and the calculated reflectivity $R$ from these optical constants are also shown in these figures. Reflectivity of zinc surfaces are shown Fig. 2(a). It can be concluded from this figure that, for a fourfold increase in effective surface area (see Table 5), the reflectivity drops by approximately $13 \%$ maintaining a similar trend as the polished surface. The reflectivity spectra of the polished samples matches well with the ellipsometry measurements (dashed curves). This indicates that the reflection is specular in nature for Zn1 and becomes more diffused for Zn2 due to micro and nano scratches from polishing. In Fig. 2(b), the reflectivity of forming steel surfaces are shown. The trend in reflectivity of polished forming steel, as function of $\lambda$, is quite the opposite of polished zinc. Generally, two kinds of oxides namely, $\alpha-\mathrm{Fe}_{2} \mathrm{O}_{3}$ (hematite) and $\mathrm{Fe}_{3} \mathrm{O}_{4}$ (magnetite), are formed over low carbon steel samples in layered structures [28, 29]. After pickling, the samples start to rust and, over time, a visual coloration of oxides are observed [29]. Therefore, in Fig. 2(b), the reflectivity of two surfaces with the same surface roughness are shown. A clean sample shows more reflectivity than the oxidized sample, which is in agreement with the literature [28]. For comparison, the reflectivity $R$ calculated from $n$ and $k$ of $\mathrm{Fe}$ [27], 
carbon steel [30], hematite and magnetite [29] are also shown in Fig. 2(b). The polished forming steel sample shows high reflectivity and follows a similar trend as Fe and carbon steel. Fig. 2(c) shows the reflectivity $R$ of galvanized steel samples. The effect of coating thickness reduction during surface preparation is easily observed. The polished surface resembles with the ellipsometry measurement, indicating specular reflectivity. With increasing roughness, the reflectivity drops, except for as received galvanized steel sample (GI3). For shorter wavelengths, GI3 reflects more than the polished sample (GI1). Since GI3 surfaces are platykurtic (see Supplementary material Fig. S.1), this indicates an increased specular reflection from the surface due to fewer "hills" and "valleys" over the surface. In Fig. 2(d), a Fresnel plot calculated from $n$ and $k$ of different metals at laser wavelength of 515 and $1030 \mathrm{~nm}$ is shown for $p$-polarized light. All the curves show an absorption maxima around an angle of incidence of $75^{\circ}-85^{\circ}$. This means that absorption will be considerably higher at local surface points where the transition between local peaks and valleys are steep. In line with the experiments, 1D ray tracing simulation also suggests that reflection is dominantly specular for surfaces with a small RMS slope (see Supplementary material Fig. S.3). This specular nature of reflection broadens with increasing surface roughness in a similar manner as IAD, which indicates more diffuse reflection. Therefore, once a stable crater is formed, the slope of the crater acts as the surface inclination angle and reduces the effect of initial surface roughness (see Supplementary material Fig. S.4).

\subsection{Crater morphology}

In order to investigate the effect on different surfaces on the laser-induced craters, the morphologies of the laser ablated craters are analyzed using SEM. The crater morphology depends on the applied laser fluence level and the number of laser pulses on the same location. Typically, the crater diameter and depth increase with increasing fluence and/or number of pulses. Since surface impurities, adsorbates and oxides are present on the sample surfaces, the morphology of the craters processed with single pulse are more affected due to these surface conditions than craters which are the result of more pulses. Fig. 3 shows the SEM images of laser irradiated surfaces of the different samples with low and high initial surface roughness for single laser pulse $(N=1)$ and a peak fluence of $F_{0}=2.1 \mathrm{~J} / \mathrm{cm}^{2}$ at $1030 \mathrm{~nm}$ laser wavelength. As can be observed from Fig. 3 for galvanized steel and zinc, crater diameter decreases with increasing roughness, which indicates that threshold fluence for detectable surface modification increases with increasing $R_{a}$ (see also Supplementary material Fig. S.8). Furthermore, the exact boundary between the processed and unprocessed area becomes increasingly difficult to distinguish for low number of pulses for increasing initial surface roughness. The presence of scratches on the surface prior to processing results in higher scattering and/or absorption of the laser beam. That is because the surface modification happens outside the irradiated zone along the scratches, see Fig. 3(a). It can also be observed that, with increasing roughness, the crater becomes more melt dominated. For the forming steel sample, the crater diameter increases with increasing roughness, see Fig. 3(c) and 3(d). The white spots in Fig. 3(c) are due to dust particles. On the rougher sample, periodic melt structures form over the crater area along the rolling scratches, whereas on the smoother 
sample, the crater appears to be a clean modified area. A similar morphology of forming steel samples is observed at a laser wavelength of $515 \mathrm{~nm}$ (as shown in Supplementary material Fig. S.5(a) and (b)).
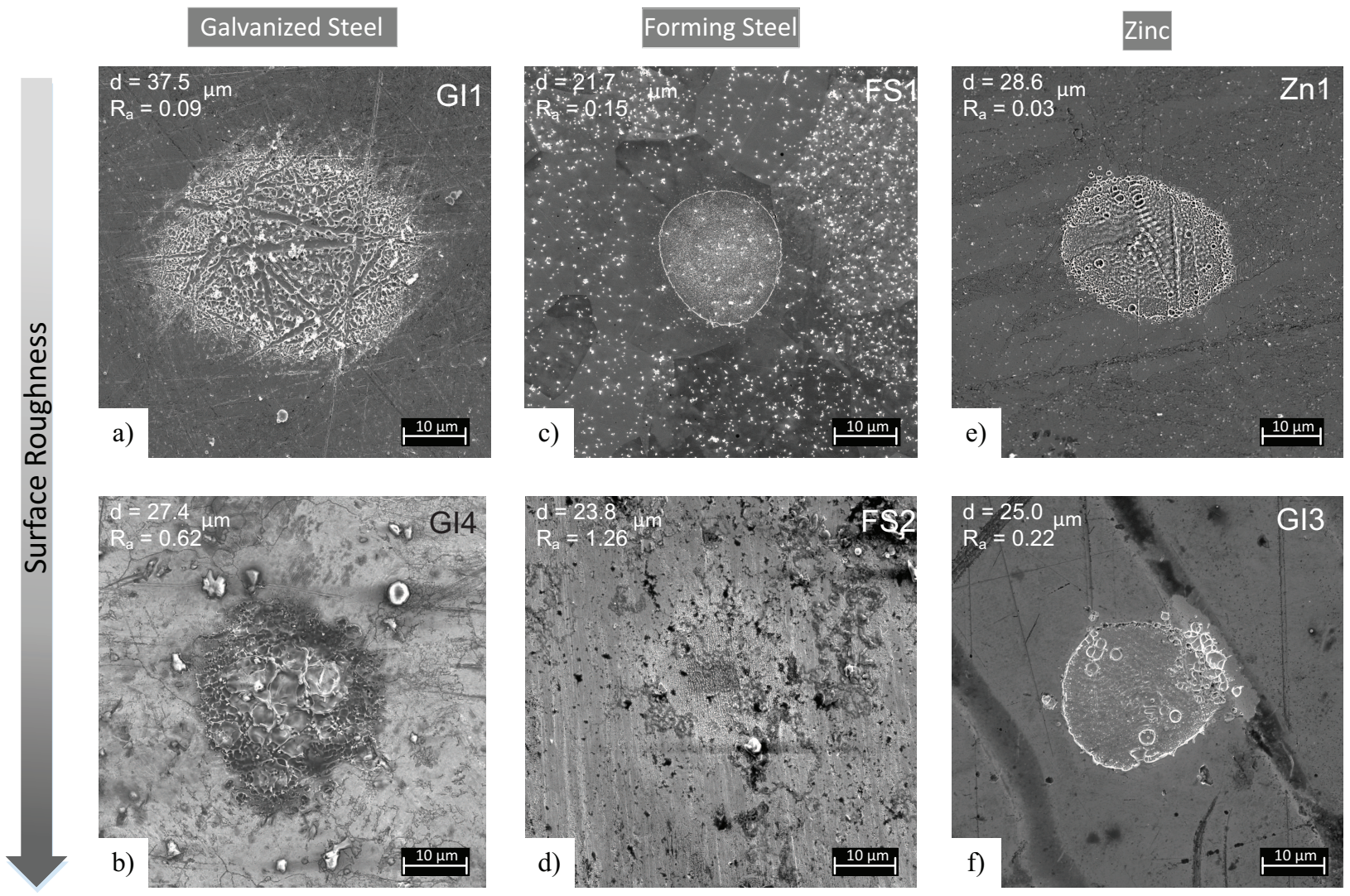

Figure 3: SEM images (top view) of samples irradiated with a single pulse at a peak fluence of $F_{0}=2.1 \mathrm{~J} / \mathrm{cm}^{2}$ and a wavelength of $1030 \mathrm{~nm}$. Diameter, $d$ of the modified surface and average surface roughness, $R_{a}$ of the unprocessed surface are derived from CLSM measurements. All images are in the same scale.

It was found that the dimensional increase of the craters becomes material dependent with multiple laser pulses. Shapes of the craters in both zinc and galvanized steel are Gaussian in nature and become increasingly melt dominated with increasing fluence and/or number of pulses (see Supplementary material Fig. S.6). This deviation from the Gaussian shape happens as the complete zinc layer, within the laser irradiated area, is ablated from galvanized steel and the steel is "exposed". Zinc surfaces get more melt dominated and similar craters was also observed in 31. For forming steel, multipulse irradiation at $F_{0}>10 \mathrm{~J} / \mathrm{cm}^{2}$ results in microcapillary channels similar to the "random walk of the drilling laser beam" as reported in [32]. For $F_{0}<10 \mathrm{~J} / \mathrm{cm}^{2}$, increasing number of pulses results in laser-induced periodic surface structures (LIPSS) formation [33, 34] as shown in Fig. 4. LIPSS appear on forming steel when processed, either with a wavelength of $1030 \mathrm{~nm}$ or 515 $\mathrm{nm}$, and for 5 to 50 pulses processing the spot. For a wavelength of $1030 \mathrm{~nm}$, high spatial 


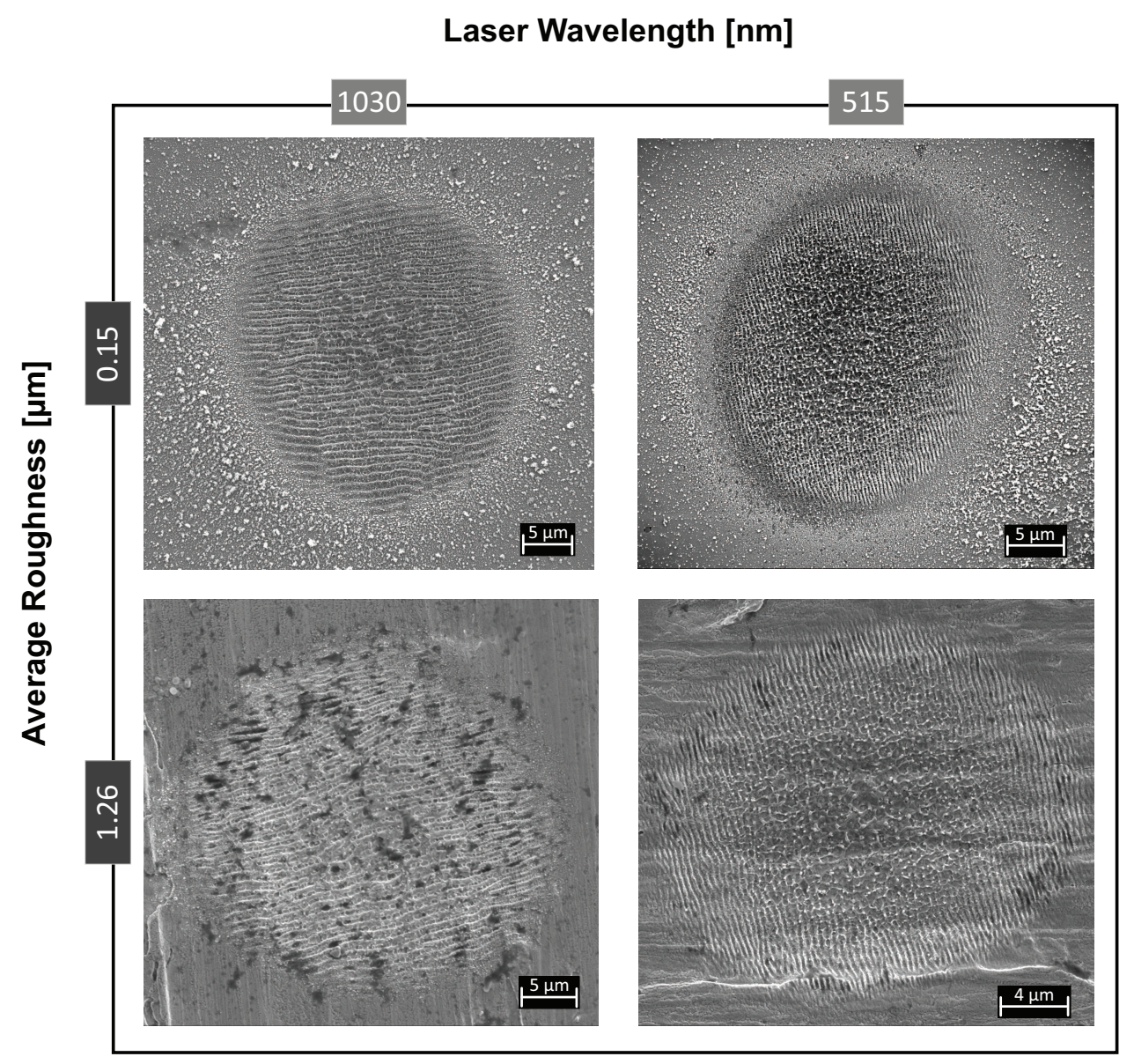

Figure 4: SEM micrographs of LIPSS on laser processed forming steel surface at $N=50, F_{0}=2.1 \mathrm{~J} / \mathrm{cm}^{2}$ at two different laser wavelengths and surface roughnesses.

frequency LIPSS (HSFL) [35] are found after 5 subsequent pulses parallel to the $E$-field of the laser polarization direction with a periodicity of $360 \pm 54 \mathrm{~nm}$. On the contrary, the HSFL periodicity could not be determined accurately since the HSFL transit smoothly into grooves [36] when processed with a wavelength of $515 \mathrm{~nm}$. Low spatial frequency LIPSS (LSFL) 35] start to form after 10 subsequent pulses at the same spot perpendicular to the direction of the E-field of the laser polarization and "erase" the HSFL. The periodicity of the LSFL decreases with increasing number of pulses from $925 \pm 20 \mathrm{~nm}$ for $\mathrm{N}=10$ to $840 \pm 90$ $\mathrm{nm}$ for $N=50$ when processed with a wavelength of $1030 \mathrm{~nm}$ and for a wavelength of 515 $\mathrm{nm}$ from $440 \pm 55 \mathrm{~nm}$ to $380 \pm 20 \mathrm{~nm}$. This results are consistent with literature [34, 35, 37]. On $\mathrm{Zn}$ on the other hand, polarization direction independent LSFL appear at one pulse per spot at a wavelength of $1030 \mathrm{~nm}$ and peak fluence levels up to $3 \mathrm{~J} / \mathrm{cm}^{2}$ at surface defects on the sample surface (see Supplementary material Fig. S.7). Here, surface defects initialize excitations of surface plasmon polaritons (SPP), which interfere with the incoming laser beam and form LSFL [36, 38. But since the melting threshold of $\mathrm{Zn}$ is below the melting threshold of steel (see Table 4), LIPSS on Zn will be "destroyed" due to melting at lower 
fluence levels and less pulses per spot than on steel. Occurrence of LIPSS on different sample at different processing conditions is summarized in Table 1 of the supplementary document.

\subsection{Ablation threshold}

Several methods have been used in the literature for determining the fluence ablation threshold of materials from the geometric features of the ablated crater, namely diameter [39, 40], depth [41, 42] and volume [43 45]. Among these parameters, the "volume method" is considered to result in the most accurate threshold determination [31. However, determining the crater depth and the ablated volume, with respect to the reference surface, becomes increasingly error-prone with increasing surface roughness. That is, as the initial surface roughness increases, determining the diameter of the laser ablated zone is relatively more accurate than depth and volume. This is an additional benefit of the $D^{2}$ - method to the benefits mentioned in [31]. Therefore, we employed the most widely used $D^{2}$ - method [14, 22, 39, 40, 42, 46,49] to determine the ablation threshold of materials at difference surface roughness values.

(a) $\times 10^{3}$

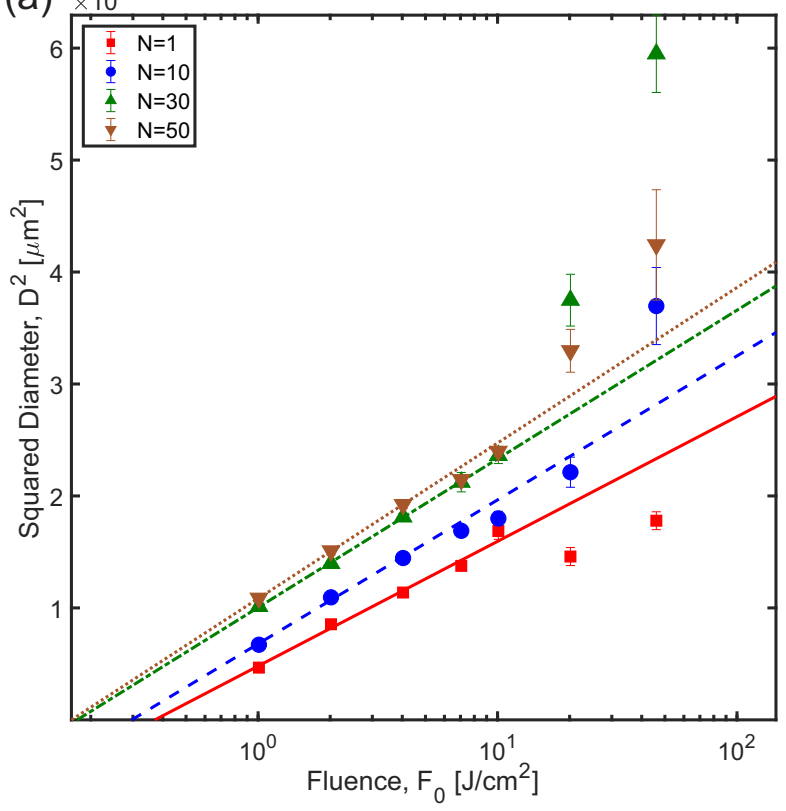

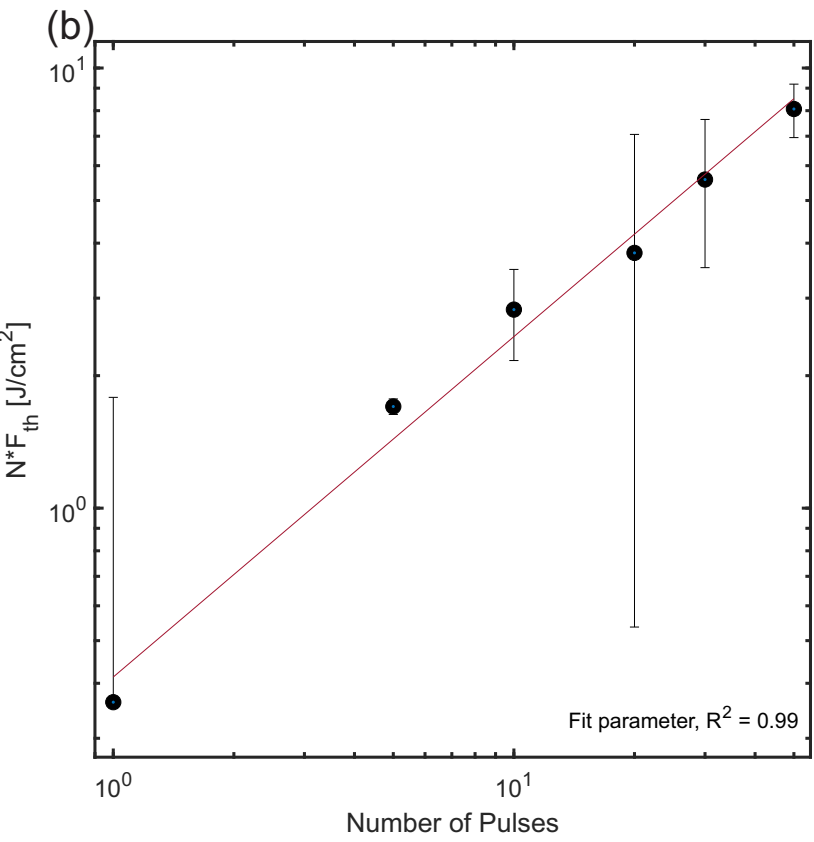

Figure 5: (a) Squared diameter $D^{2}$ of the ablated crater for different number of pulses $N$ as a function of the peak laser fluence $F_{0}$ for forming steel sample (FS1) with $R_{a}=0.15 \mu \mathrm{m}$. The linear curves represent least squared fits according to Eq. (1). (b) Accumulation in the fluence ablation threshold,$N \cdot F_{t h}(N)$ as a function of laser pulse number $N$. The solid curve represents a least squared fit according to Eq. (2).

Given that the laser fluence profile is Gaussian shaped, the ablated crater diameter $D$ is 
related to the laser peak fluence $F_{0}$ and the ablation fluence threshold $F_{t h}$ as [39]

$$
D^{2}=2 \omega_{0}^{2} \ln \left(\frac{F_{0}}{F_{t h}}\right)
$$

If the squared diameter $D^{2}$ measured from the confocal data is plotted as a function of peak fluence $F_{0}$ in a semi-logarithmic graph, the fluence threshold $F_{t h}$ can be determined from the intersection of the linear extrapolation of the fitted curve with the horizontal axis, so at $D^{2}=0$. This graph can also be used to determine the beam diameter from the slope of the curves [39]. However, the beam diameter calculated from the slope does not necessarily reflect the actual beam diameter, due to the change in absorptivity within the irradiated zone after multiple pulses [49, 50]. In Fig. 5(a), the squared diameters $D^{2}$ of ablated craters on forming steel sample (FS1) is plotted against the peak fluence for different number of pulses. In this figure, each data point is based on a minimum of 9 craters up to a maximum value of 15 craters. The observed nonlinearity at high peak fluence values is due to the surface modification by the adequately intense "tails" of Gaussian intensity profile of the laser beam at higher fluence levels. Extrapolating the fitted curve to $D^{2}=0$ results in $F_{t h}$ for a given number of laser pulses. It is apparent from Fig. 5 that the threshold fluence decreases as the applied number of pulses increases, which indicates an accumulative behavior, i.e. incubation. To account for this incubation behavior, a power law relating the ablation threshold fluence $F_{t h}(N)$ for $N$ pulses to single pulse $F_{t h}(1)$ through the incubation coefficient $\zeta$ as exponent, is given by [51]

$$
N \cdot F_{t h}(N)=F_{t h}(1) \cdot N^{\zeta}
$$

Threshold fluences resulting from the extrapolation of the fitted curves according to Eq. (1) (see Fig. 5(a)) are plotted against the number of laser pulses in Fig. 5(b). That is, the incubation in threshold fluences, i.e. $N \cdot F_{t h}(N)$, is shown as a function of $N$ and a nonlinear least-square fit along the data points is plotted according to Eq. (2) in Fig. 5(b). The fit results in a single pulse threshold fluence of $0.55 \pm 0.09 \mathrm{~J} / \mathrm{cm}^{2}$ and an incubation coefficient of $0.66 \pm 0.18$ with a $R^{2}$ value of $98.7 \%$. Similar calculations were performed for other samples mentioned in Table 5. The corresponding threshold fluences and incubation coefficients for all the samples are shown in Fig. 6 and 7 respectively.

As mentioned in Sec. 3.3, apart from initial surface roughness, the initial surface conditions, such as oxides, affect the ablation threshold for $N=1$. In multi-pulse irradiation, the energy of the first laser pulse contributes not only to material ablation, but also to surface "cleaning" [12, 31. Therefore, $F_{t h}(1)$ from Eq. (1) for $N=1$ and Eq. (2) differs depending on the initial surface condition of the sample. This can be observed in Fig. 5(b), where the estimated threshold fluence from the fit of Eq. (2) is greater than $F_{t h}$ for $N=1$. For this reason, in the following, single pulse threshold fluence from Eq. (1) and (2) will be referred to as $F_{t h}(1)$ and $F_{t h}(\zeta)$ respectively. In Fig. 6, both $F_{t h}(1)$ and $F_{t h}(\zeta)$ are shown for all the samples under consideration against the increase in actual surface area $(S A)$ to projected area $(A), \Delta_{S A / A}$. In Fig. 6(a), threshold fluences calculated from the fit of Eq. (1) and (2), namely $F_{t h}(1)$ and $F_{t h}(\zeta)$, are shown for galvanized steel samples processed at a wavelength 

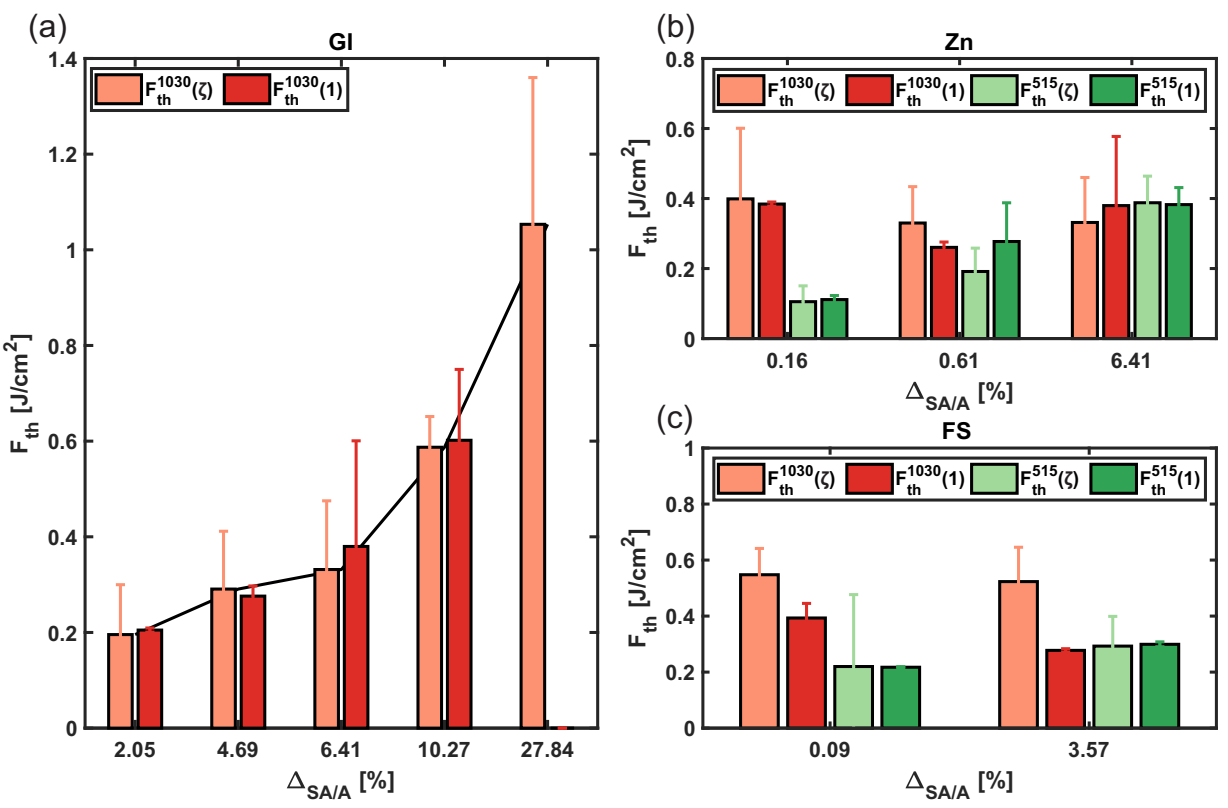

Figure 6: Threshold fluences $F_{t h}(1)$ and $F_{t h}(\zeta)$ as a function of increase in surface area $\Delta_{S A / A}$ for (a) galvanized steel (GI), (b) zinc (Zn) and (c) forming steel (FS).

of $1030 \mathrm{~nm}$. For GI5, the craters for $N=1$ were not observable under optical microscope. Therefore, only $F_{t h}(\zeta)$ is plotted for GI5 in Fig. 6(a). In this figure, both $F_{t h}(1)$ and $F_{t h}(\zeta)$ increase linearly with the increase in surface area as $0.03 \cdot\left(\Delta_{S A / A}\right)+0.13$. Hence, it can be concluded that the threshold fluence increases with the increase in effective surface area for galvanized steel at $1030 \mathrm{~nm}$. However, this is not the case for zinc and forming steel samples, processed at a wavelength of $1030 \mathrm{~nm}$, as shown in Fig. 6(b) and (c) respectively. For zinc, $F_{t h}$ at a wavelength of $1030 \mathrm{~nm}$ first decreases with increasing $\Delta_{S A / A}$ with a negative slope of 0.115 and then increases again as shown in Fig. 6(b). This trend is more pronounced for $F_{t h}^{1030}(1)$ than $F_{t h}^{1030}(\zeta)$ (see Fig. 6(b)). On the other hand, threshold fluence of forming steel decreases for increasing $\Delta_{S A / A}$ with a negative slope of 0.009 as shown in Fig. 6(c). Again, the trend is more pronounced for $F_{t h}^{1030}(1)$ and $F_{t h}^{1030}(\zeta)$ stays nearly constant. Since the correlation between the increase in effective surface area and the threshold fluences of $\mathrm{Zn}$ and FS at a wavelength of $1030 \mathrm{~nm}$ is weak, we performed ablation experiment on Zn and FS samples at a wavelength of $515 \mathrm{~nm}$. In this case. the aspect ratio between the surface features and the laser wavelength of $515 \mathrm{~nm}$ increases by twofold than of $1030 \mathrm{~nm}$. As can be seen in Fig. 6 (b) and (c), both $F_{t h}^{515}(1)$ and $F_{t h}^{515}(\zeta)$ increases with increasing roughness with a positive slope of 0.144 and 0.026 for $\mathrm{Zn}$ and forming steel respectively. Also, for a decrease in laser wavelength by half, the threshold fluence reduces by half for smooth surfaces, and by a quarter for rough surfaces (see Fig. 6 (b) and (c)).

Incubation coefficients $\zeta$ resulting from the fit of Eq. (2) are shown in Fig. 7. Incubation coefficient indicates the degree of damage accumulation within the irradiated area with respect to material removal by multiple laser pulses. The incubation coefficient is less than 1 if the accumulated damage (i.e. crystal defects, lattice strains, heat accumulation etc) is 

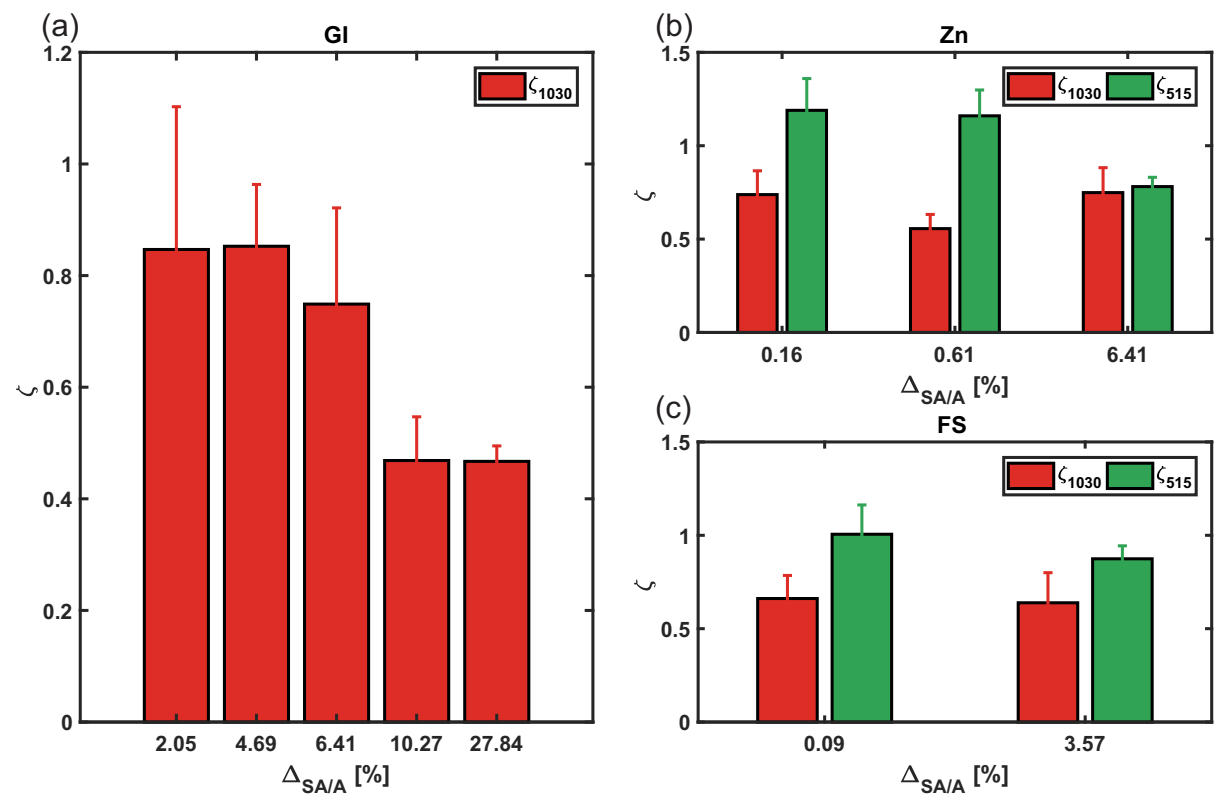

Figure 7: Incubation coefficients $\zeta$ as a function of increase in surface area $\Delta_{S A / A}$ for (a) galvanized steel (GI), (b) zinc (Zn) and (c) forming steel (FS).

conducive to material removal by the subsequent laser pulses, whereas the incubation coefficient is greater than 1 if the damage accumulation hinders material removal [51]. In Fig. $7\left(\right.$ a),$\zeta$ decreases from about 0.8 to 0.5 with increasing $\Delta_{S A / A}$ for galvanized steel indicating higher degree of damage accumulation. For $\mathrm{Zn}$ and FS, $\zeta_{1030}$ stays almost constant while $\zeta_{515}$ decreases with increase in effective surface area. From Fig. 6 and 7, it can be concluded that, while threshold fluence $F_{t h}$ increases with the increase in effective surface area $\Delta_{S A / A}$, incubation coefficient $\zeta$ decreases with $\Delta_{S A}$, showing tendencies towards higher damage accumulation effect with increasing surface roughness. As a result, the difference in threshold fluence with increasing $\Delta_{S A / A}$ becomes smaller for $N>1$ (see Supplementary material Fig. S.9. . Moreover, a decrease of the incubation coefficient with increasing initial surface roughness is more pronounced in soft $(\mathrm{Zn})$ than relatively hard (forming steel) material.

\section{Discussion}

From the perspective of laser material processing, the effective surface area (roughness) is known to affect the absorptivity of the laser beam and, consequently, the fluence ablation threshold. Although both the absorptivity and ablation threshold depend on the laser wavelength and on the material properties, it would be interesting to see if there is any dependency of these properties on the increase in effective surface area, irrespective of laser wavelength and material, at a pulse duration of 7 ps. Figure 8 shows a scatterplot matrix with all the results of Sec. 3.2 and 3.4 in order to estimate the plausible relationships between the three variables namely, increase in effective surface area $\Delta_{S A / A}$, absorptivity 
$(A)$ and threshold fluence $F_{t h}(\zeta)$. In this figure, the variable names are shown diagonally and each of the variables is plotted against each other. The plot boxes above and below the diagonal are mirror images with inverted $\mathrm{X}$ and $\mathrm{Y}$ axis.

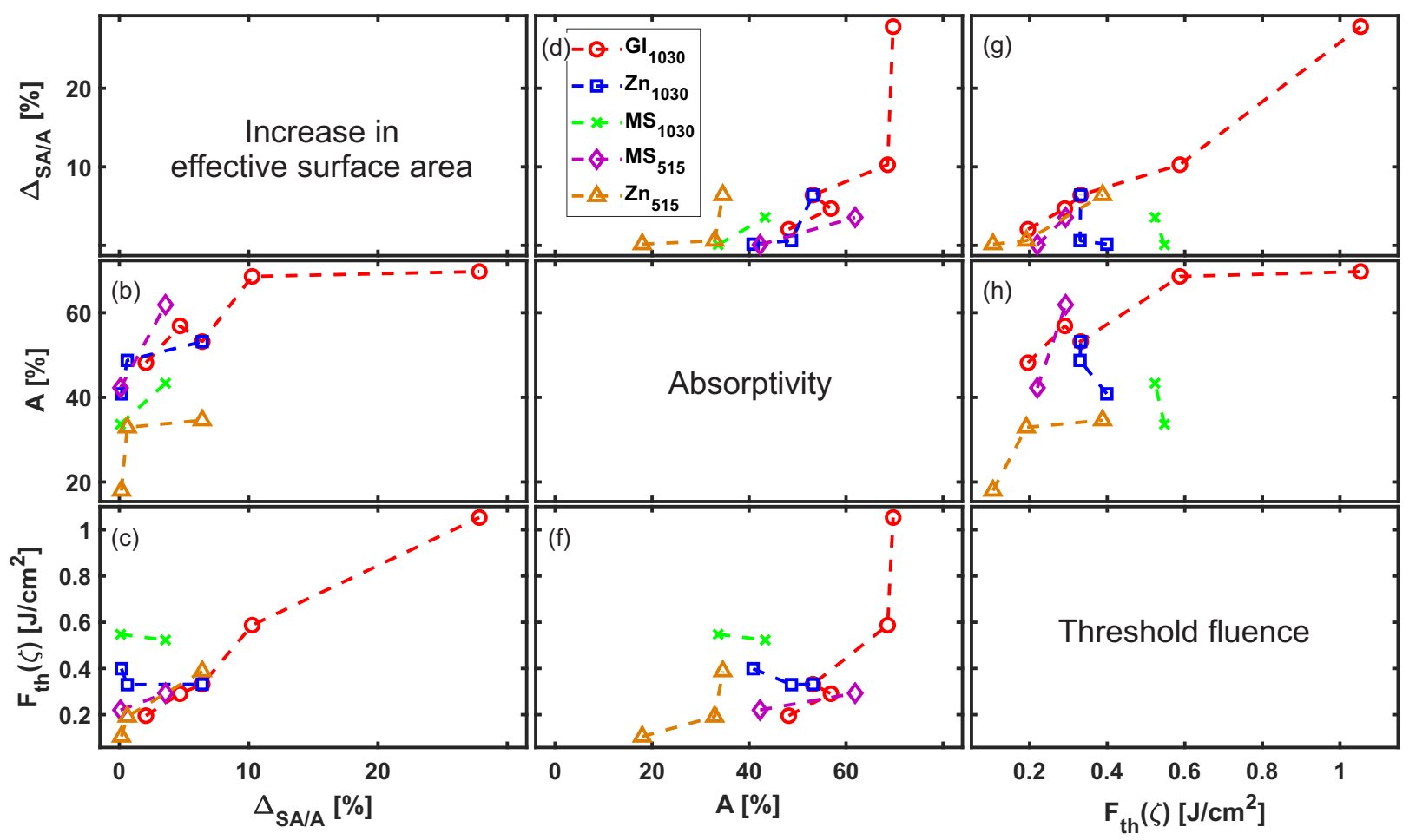

Figure 8: Scatterplot matrix, based on confocal microscopy and integrating sphere measurement data, relates increase in effective surface area $\Delta_{S A}$, absorptivity $A$ and threshold fluence $F_{t h}(\zeta)$.

In this scatterplot, there is a positive correlation between $\Delta_{S A / A}$ and $F_{t h}(\zeta)$ for most of the data points (see Fig. 8 (c) and (g)). On the other hand, $\Delta_{S A / A}$ and $A$ as well as $F_{t h}(\zeta)$ and $A$ seems to correlate exponentially (see Fig. $8(\mathrm{~b}),(\mathrm{d})$ and $(\mathrm{f}),(\mathrm{h}))$. In the context of this work, optical absorption $A$ of the surface increases with an increase in surface roughness, be it $R_{a}, \sigma, \sigma / \tau$ or $S A / A$ (see Fig. 8 (b)) and (d). This confirms the existent relationship between optical absorption and surface roughness [5, 6, 52, 53]. The presence of oxides and surface contaminants further promotes this behavior. However, may be counter-intuitively, it appears that, the threshold fluence $F_{t h}(\zeta)$ increases exponentially with the increase in absorptivity $A$ (see Fig. 8 (f) and (h)), whereas it increases linearly with the increase in surface roughness $\Delta_{S A / A}$ (see Fig. 8 (c) and (g)) for most of the data points. There lies exceptions, for example, $F_{t h}$ of zinc and forming steel at $1030 \mathrm{~nm}$ wavelength (see Fig. 6 (b) and (c)). For zinc, there is first a decrease and then an increase in threshold fluence with the increasing $\Delta_{S A / A}$ (see Fig. 6(b)). First, the decrease in threshold fluences, both $F_{t h}^{1030}(1)$ and $F_{t h}^{1030}(\zeta)$, may be attributed to the presence of micro and nano scratches on the surface (see Fig. 6 (b)). The threshold fluence for large scratches is higher than micro and nano 
scratches [14, 54]. Also the material heats up more in the peaks of the surface roughness than in the valleys, because of higher heat diffusion in the valleys [3]. Therefore, a $30 \%$ drop in $F_{t h}$ is seen as $\Delta_{S A / A}$ is increased by a factor of 4 for Zn2 compared to Zn1 (see Fig. 6 (b)). The degree of damage accumulation, i.e. incubation, is also higher for the sample with scratches (Zn2) than Zn1 (see Fig. 7(b)). Since Zn2 heats up more than Zn1, relatively less pulse energy is required to ablate. This is also reflected in multi-pulse processing (see Supplementary material Fig. S.9), where the threshold fluence of Zn2 reduces by about $50 \%$ over 10 pulses compared with $30 \%$ drop over single pulse than Zn1. Secondly, an increase in $F_{t h}(1)$ is observed for $\Delta_{S A / A}=6.41 \%$. For $N=1$, the as received galvanized steel sample $\left(R_{a}<0.3 \mu \mathrm{m}\right)$ is comparable to the pure zinc sample. This is because, optical heating is a surface phenomenon and the effect of the substrate can be neglected for a coating thickness of $8 \mathrm{\mu m}$ or larger. However, the substrate effect becomes prominent for multiple pulses. Initial surface of hot-dipped galvanized steel features dendrites. The width and depth of these dendritic arms are much larger than the micro scratches present in surfaces of Zn2 (see Sec. 3.3). As a result, the target material surface does not heat up as fast as microscratched surfaces and, consequently, an increase in $F_{t h}(1)$ as well as $\zeta$ is observed for Zn sample with $\Delta_{S A / A}=6.41 \%$ (see Fig. 6(b) and 7 (b)). In the case of forming steel, trend in $F_{t h}$ vs $\Delta_{S A / A}$ at $1030 \mathrm{~nm}$ may be attributed to an enhanced absorption by the oxides. When applying multiple pulses, this effect is overcome and $F_{t h}(\zeta)$ of the smooth and rough surface is almost equal (see Fig. 6 (c)). It can be concluded from Fig. 8 that all three parameters absorptivity, threshold fluence and effective surface area increase mutually with respect to each other. For example, an increase of $27 \%$ in effective surface area results in $45 \%$ increase in absorptivity and $380 \%$ increase in threshold fluence compared to the smooth surface for galvanized steel. Moreover, the incubation coefficient decreases with increasing surface roughness (see Fig. 7). As the surface roughness increases, an increased absorption of laser beam, resulting in higher threshold fluence and lower incubation coefficient, indicates a heat and/or defect accumulation with increasing surface roughness.

To study the increase in threshold fluence with increasing absorbed energy, we modeled the one dimensional heat accumulation behavior of Zn over multiple pulse irradiation (see Supplementary material Sec. V). For multiple pulses, the sample heats up from the residual heat of the previous pulse. The degree of heat accumulation, apart from being material-dependent property, also depends on the time between consecutive pulses on the same location. Figure 9(a) shows the simulation result of the model (see Supplementary material Eq. (2)) for $N=10$, time between pulses, $t_{p-p}=3.9 \mathrm{~ms}$, incident pulse energy $E_{p}=30 \mu \mathrm{J}$ and laser wavelength of $1030 \mathrm{~nm}$ for different surface roughnesses. As can be observed, with increasing sample roughness, sample heating increases. This is in agreement with [55, 56]. In Fig. 9(b), the threshold fluence and residual heat is plotted against increase in surface area $\left(\Delta_{S A / A}\right)$ for galvanized steel samples. In this figure, the as received GI sample $\left(\mathrm{GI} 3, \Delta_{S A / A}=6.4 \%\right)$ having low $\eta_{\text {res }}$ is due to higher reflectivity of GI3 sample, resulting from the presence of higher amount of Al on the HDG steel surface [57 59]. On the whole, an increase in residual heat and threshold fluence is positively correlated with the increase in effective surface area. 

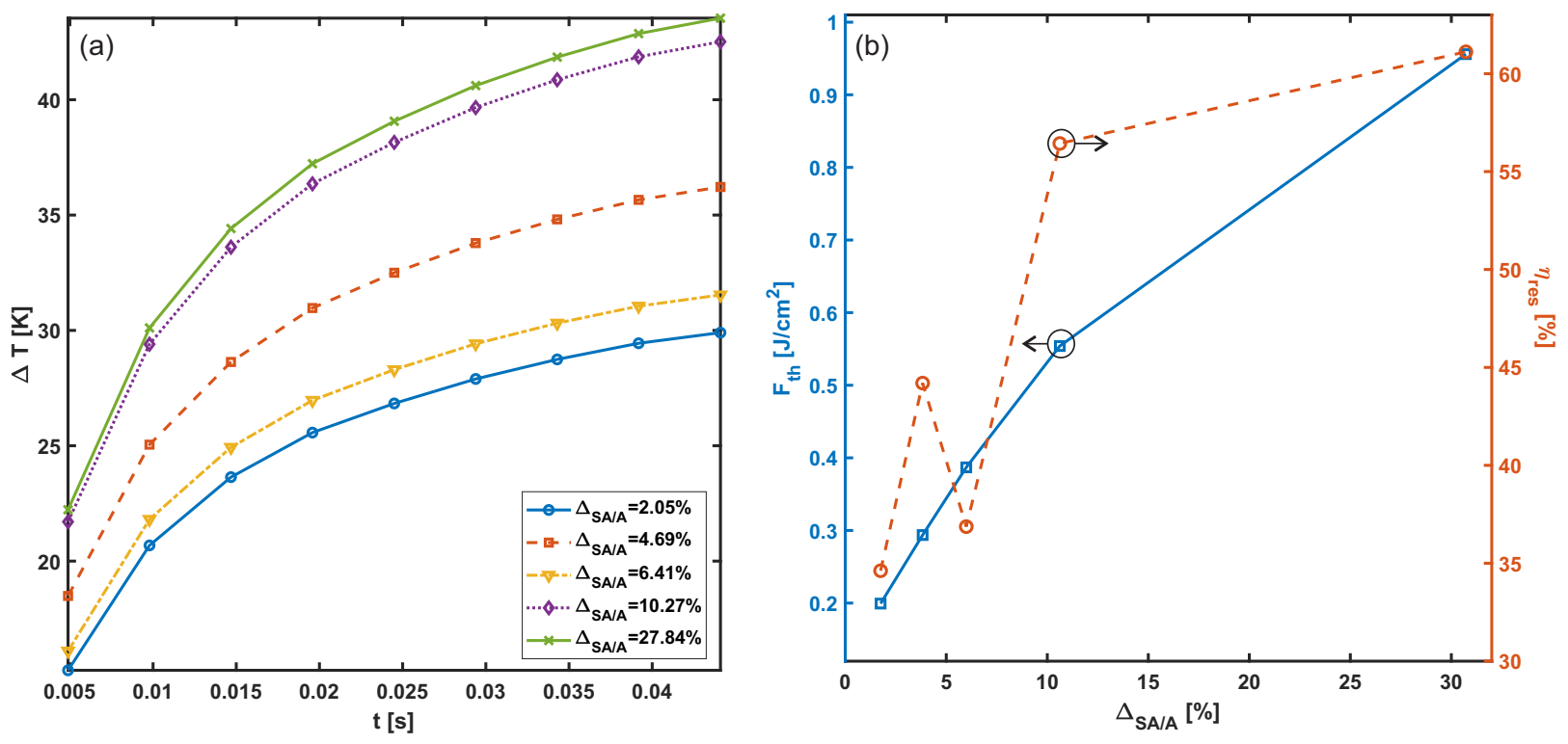

Figure 9: (a) Simulation of the difference of the maximum temperature increase induced by a stationary laser beam using $t_{p-p}=3.9 \mathrm{~ms}, \omega_{0}=14.5 \mu \mathrm{m}, E_{p}=30 \mu \mathrm{J}, N=10$ and material parameters for galvanized steel (Zn-coated) according to Table 4. (b) Evolution of threshold fluence and residual heat coefficient with increase in effective surface area for galvanized steel at a wavelength of $1030 \mathrm{~nm}$.

From the above discussions, we propose the following explanation. With increasing surface area, more energy is required to ablate materials. This extra energy requirement is expected to be met by increased absorption. During ultrashort pulse processing, a thin surface layer is heated up to a nonuniform temperature distribution due to surface irregularities. For thin films, the surface roughness locally increases sheet resistance through $e^{-}$scattering [60]. As a result, preferential ablation becomes dominant as roughness increases. Rather than ablating the material, most of the absorbed energy ends up as residual heat. This observation is further confirmed from Fig. 3, more melt dominated structures are visible for higher roughness samples. When applying multiple pulses, residual heat leads to a higher degree of incubation. Therefore, for a given material, the difference between multiple-pulse threshold fluences $\left(F_{t h}(N)\right)$ for different surface roughnesses becomes less pronounced than the difference between single-pulse threshold fluences $\left(F_{t h}(1)\right)$.

\section{Conclusion}

Zinc, galvanized steel and forming steel surfaces showing different roughness characteristics were investigated for picosecond pulsed laser ablation at wavelengths of 515 and 1030 $\mathrm{nm}$. A close look at the surfaces revealed that the average roughness value $R_{a}$ does not properly describe the different kinds of surface features present on the surfaces from the framework of absorption of laser energy. That is because these features lead to different local angle of incidence for a normally incident beam, as well as an increase in effective surface 
area. Reflectivity measurements of the surfaces showed increased absorption with increasing effective surface area, which is in agreement with the existing literature. However, threshold energy requirement for laser ablation is also found to increase with increasing effective surface area. The presence of surface impurities and oxidation may reverse this effect in the case of single pulse processing. Decreasing laser wavelength by half results in a proportional decrease in threshold fluence, while the rate of decline is higher for rough surfaces than smooth surfaces. From a 1D ray tracing simulation, the scattering angle is found to increase with increasing effective surface area which leads to a nonuniform temperature distribution due to the absorbed laser energy. A heat accumulation model showed that, as the effective surface area increases, most of the absorbed energy is left as residual heat in the laser irradiated zone. For multiple laser pulses, this leads to higher degree of incubation for surfaces with larger effective surface area. Therefore, when surface roughness is concerned, increased laser absorption does not necessarily imply increased material ablation if the number of laser pulses is low. For high ablation efficiency with low number of pulses, the initial surface roughness should be low.

\section{Acknowledgments}

The authors would like to acknowledge the financial support of Tata Steel Nederland Technology BV. We would also like to thank Dr. B. Pathiraj of the University of Twente for his fruitful discussions on this topic and R. Roos, R.J. van Dasselaar, R.M. Reef, M. Veugelers, K.J. Smelt, W. R. Pot, S.J. van Haaren and R.C. Gerritsen for their help with the experimental work.

\section{References}

[1] D. Zhang, L. Guan, Comprehensive Materials Processing, Vol. 4, Elsevier, 2014, Ch. Laser Ablation.

[2] H. Costa, I. Hutchings, Some innovative surface texturing techniques for tribological purposes, Proceedings of the Institution of Mechanical Engineers, Part J: Journal of Engineering Tribology 229 (4) (2015) 429-448.

[3] D. Bäuerle, Laser Processing and Chemistry, Springer Berlin Heidelberg, 2011.

[4] D. Bergström, The absorption of laser light by rough metal surfaces, Ph.D. thesis, Luleå Tekniska Universitet (2008).

[5] M. Auinger, P. Ebbinghaus, A. Blümich, A. Erbe, Effect of surface roughness on optical heating of metals, Journal of the European Optical Society-Rapid publications 9.

[6] J. Xie, A. Kar, Laser welding of thin sheet steel with surface oxidation, Welding Journal 78 (1999) $343-\mathrm{s}$.

[7] T. Wieting, J. DeRosa, Effects of surface condition on the infrared absorptivity of 304 stainless steel, Journal of Applied Physics 50 (2) (1979) 1071-1078.

[8] M. Sokolov, A. Salminen, Methods for improving laser beam welding efficiency, Physics Procedia 56 (2014) 450-457.

[9] J.-T. Wang, C.-I. Weng, J.-G. Chang, C.-C. Hwang, The influence of temperature and surface conditions on surface absorptivity in laser surface treatment, Journal of Applied Physics 87 (7) (2000) 3245-3253.

[10] L. V. Zhigilei, Z. Lin, D. S. Ivanov, Atomistic modeling of short pulse laser ablation of metals: connections between melting, spallation, and phase explosion, The Journal of Physical Chemistry C 113 (27) (2009) 11892-11906. 
[11] A. Perrone, L. Cultrera, A. Dima, D. Guido, A. Zocco, J. C. Conde, P. González, J. Serra, B. Léon, Extensive studies of the plume deflection angle during laser ablation of Si target, Japanese Journal of Applied Physics 42 (7R) (2003) 4181.

[12] A. Vorobyev, C. Guo, Reflection of femtosecond laser light in multipulse ablation of metals, Journal of Applied Physics 110 (4) (2011) 043102.

[13] M. R. B. Shahadat, A. S. Masnoon, S. Ahmed, A. K. M. M. Morshed, Effect of surface roughness and void fraction on thermal transportation of a solid: a molecular dynamics study, in: AIP Conference Proceedings, Vol. 1919, AIP Publishing, 2017, p. 020037.

[14] B. Wu, P. Liu, X. Wang, F. Zhang, L. Deng, J. Duan, X. Zeng, Effect of laser absorption on picosecond laser ablation of $\mathrm{Cr} 12 \mathrm{MoV}$ mold steel, $9 \mathrm{Cr} 18$ stainless steel and H13A cemented carbide, Optics \& Laser Technology 101 (2018) 11-20.

[15] I. Lopez-Quintas, V. Pinon, M. P. Mateo, G. Nicolas, Effect of surface topography in the generation of chemical maps by laser-induced plasma spectroscopy, Applied Surface Science 258 (23) (2012) 94329436.

[16] A. Perrone, A. Zocco, L. Cultrera, D. Guido, Detailed studies of the plume deflection effect during long laser irradiation of solid targets, Applied Surface Science 197 (2002) 251-256.

[17] J. Conde, F. Lusquinos, P. Gonzalez, J. Serra, B. León, A. Dima, L. Cultrera, D. Guido, A. Zocco, A. Perrone, Finite element analysis of the initial stages of the laser ablation process, Thin Solid Films 453 (2004) 323-327.

[18] R. House, J. Bettis, A. Guenther, Surface roughness and laser damage threshold, IEEE Journal of Quantum Electronics 13 (5) (1977) 361-363.

[19] S. Babu, Surface roughness dependence of laser induced damage threshold, IEEE Journal of Quantum Electronics 15 (7) (1979) 533-535.

[20] F. C. Porter, Zinc handbook: Properties, Processing, and Use in design, CRC Press, 1991.

[21] C. Edupack, Granta design limited, Cambridge, England, UK.

[22] A. Garcia-Giron, J. Romano, Y. Liang, B. Dashtbozorg, H. Dong, P. Penchev, S. Dimov, Combined surface hardening and laser patterning approach for functionalising stainless steel surfaces, Applied Surface Science 439 (2018) 516-524.

[23] V. D. Ta, A. Dunn, T. J. Wasley, J. Li, R. W. Kay, J. Stringer, P. J. Smith, E. Esenturk, C. Connaughton, J. D. Shephard, Laser textured surface gradients, Applied Surface Science 371 (2016) 583589.

[24] M. Butt, D. Ali, M. U. Tanveer, S. Naseem, Surface roughness and electrical resistivity of high-purity zinc irradiated with nanosecond visible laser pulses, Applied Surface Science 305 (2014) 466-473.

[25] B. Bhushan, Surface roughness analysis and measurement techniques, in: Modern Tribology Handbook, CRC press, 2000, pp. 74-144.

[26] E. Gadelmawla, M. Koura, T. Maksoud, I. Elewa, H. Soliman, Roughness parameters, Journal of Materials Processing Technology 123 (1) (2002) 133-145.

[27] P. B. Johnson, R. W. Christy, Optical constants of transition metals: Ti, V, Cr, Mn, Fe, Co, Ni, and Pd, Physical Review B 9 (12) (1974) 5056.

[28] I. Iordanova, V. Antonov, Surface oxidation of low carbon steel during laser treatment, its dependence on the initial microstructure and influence on the laser energy absorption, Thin Solid Films 516 (21) (2008) $7475-7481$.

[29] V. Goossens, J. Wielant, S. Van Gils, R. Finsy, H. Terryn, Optical properties of thin iron oxide films on steel, Surface and Interface Analysis: An International Journal devoted to the development and application of techniques for the analysis of surfaces, interfaces and thin films 38 (4) (2006) 489-493.

[30] L. Li, The advances and characteristics of high-power diode laser materials processing, Optics and Lasers in Engineering 34 (4-6) (2000) 231-253.

[31] H. Mustafa, R. Pohl, T. C. Bor, B. Pathiraj, D. T. A. Matthews, G. R. B. E. Römer, Picosecond-pulsed laser ablation of zinc: crater morphology and comparison of methods to determine ablation threshold, Optics Express 26 (14) (2018) 18664-18683.

[32] T. Anthony, The random walk of a drilling laser beam, Journal of Applied Physics 51 (2) (1980) 
1170-1175.

[33] J. V. Obona, V. Ocelík, J. Z. P. Skolski, V. S. Mitko, G. R. B. E. Römer, A. J. Huis, J. T. M. De Hosson, On the surface topography of ultrashort laser pulse treated steel surfaces, Applied Surface Science 258 (4) (2011) 1555-1560.

[34] B. Wu, M. Zhou, J. Li, X. Ye, G. Li, L. Cai, Superhydrophobic surfaces fabricated by microstructuring of stainless steel using a femtosecond laser, Applied Surface Science 256 (1) (2009) 61-66.

[35] J. Bonse, S. V. Kirner, S. Höhm, N. Epperlein, D. Spaltmann, A. Rosenfeld, J. Krüger, Applications of laser-induced periodic surface structures (LIPSS), in: Laser-based Micro-and Nanoprocessing XI, Vol. 10092, International Society for Optics and Photonics, 2017, p. 100920N.

[36] J. Bonse, J. Krüger, Pulse number dependence of laser-induced periodic surface structures for femtosecond laser irradiation of silicon, Journal of Applied Physics 108 (3) (2010) 034903.

[37] A. Vorobyev, V. Makin, C. Guo, Periodic ordering of random surface nanostructures induced by femtosecond laser pulses on metals, Journal of Applied Physics 101 (3) (2007) 034903.

[38] J. Bonse, A. Rosenfeld, J. Krüger, On the role of surface plasmon polaritons in the formation of laserinduced periodic surface structures upon irradiation of silicon by femtosecond-laser pulses, Journal of Applied Physics 106 (10) (2009) 104910.

[39] J. M. Liu, Simple technique for measurements of pulsed Gaussian-beam spot sizes, Optics Letters 7 (5) (1982) 196-198.

[40] P. T. Mannion, J. Magee, E. Coyne, G. M. O'connor, T. J. Glynn, The effect of damage accumulation behaviour on ablation thresholds and damage morphology in ultrafast laser micro-machining of common metals in air, Applied Surface Science 233 (1) (2004) 275-287.

[41] S. Preuss, A. Demchuk, M. Stuke, Sub-picosecond UV laser ablation of metals, Applied Physics A: Materials Science \& Processing 61 (1) (1995) 33-37.

[42] M. Hashida, A. F. Semerok, O. Gobert, G. Petite, J.-F. Wagner, Ablation thresholds of metals with femtosecond laser pulses, in: Nonresonant Laser-Matter Interaction (NLMI-10), International Society for Optics and Photonics, 2001, pp. 178-185.

[43] G. Raciukaitis, M. Brikas, P. Gecys, M. Gedvilas, Accumulation effects in laser ablation of metals with high-repetition-rate lasers, in: High-Power Laser Ablation 2008, International Society for Optics and Photonics, 2008, pp. 70052L-70052L.

[44] B. Neuenschwander, B. Jaeggi, M. Schmid, G. Hennig, Surface structuring with ultra-short laser pulses: basics, limitations and needs for high throughput, Physics Procedia 56 (2014) 1047-1058.

[45] B. Neuenschwander, B. Jaeggi, M. Schmid, V. Rouffiange, P.-E. Martin, Optimization of the volume ablation rate for metals at different laser pulse-durations from ps to fs, in: SPIE LASE, International Society for Optics and Photonics, 2012, pp. 824307-824307.

[46] J. Byskov-Nielsen, J.-M. Savolainen, M. S. Christensen, P. Balling, Ultra-short pulse laser ablation of metals: threshold fluence, incubation coefficient and ablation rates, Applied Physics A: Materials Science \& Processing 101 (1) (2010) 97-101.

[47] C. W. Cheng, S. Y. Wang, K. P. Chang, J. K. Chen, Femtosecond laser ablation of copper at high laser fluence: modeling and experimental comparison, Applied Surface Science 361 (2016) 41-48.

[48] N. Lasemi, U. Pacher, L. Zhigilei, O. Bomatí-Miguel, R. Lahoz, W. Kautek, Pulsed laser ablation and incubation of nickel, iron and tungsten in liquids and air, Applied Surface Science 433 (2018) 772-779.

[49] Z. Sun, M. Lenzner, W. Rudolph, Generic incubation law for laser damage and ablation thresholds, Journal of Applied Physics 117 (7) (2015) 073102.

[50] F. Di Niso, C. Gaudiuso, T. Sibillano, F. P. Mezzapesa, A. Ancona, P. M. Lugarà, Role of heat accumulation on the incubation effect in multi-shot laser ablation of stainless steel at high repetition rates, Optics Express 22 (10) (2014) 12200-12210.

[51] Y. Jee, M. F. Becker, R. M. Walser, Laser-induced damage on single-crystal metal surfaces, Journal of the Optical Society of America B 5 (3) (1988) 648-659.

[52] D. Bergström, J. Powell, A. F. H. Kaplan, A ray-tracing analysis of the absorption of light by smooth and rough metal surfaces, Journal of Applied Physics 101 (11) (2007) 113504.

[53] D. Bergström, J. Powell, A. Kaplan, The absorption of light by rough metal surfaces-a three- 
dimensional ray-tracing analysis, Journal of Applied Physics 103 (10) (2008) 103515.

[54] P. Bizi-Bandoki, S. Valette, E. Audouard, S. Benayoun, Effect of stationary femtosecond laser irradiation on substructures' formation on a mold stainless steel surface, Applied Surface Science 270 (2013) $197-204$.

[55] A. Y. Vorobyev, C. Guo, Thermal response and optical absorptance of metals under femtosecond laser irradiation, Natural Science 3 (06) (2011) 488-495.

[56] M. Cerchez, M. Swantusch, M. Toncian, X. M. Zhu, R. Prasad, T. Toncian, C. Rödel, O. Jäckel, G. G. Paulus, A. A. Andreev, Enhanced energy absorption of high intensity laser pulses by targets of modulated surface, Applied Physics Letters 112 (22) (2018) 221103.

[57] S. Feliu Jr, V. Barranco, XPS study of the surface chemistry of conventional hot-dip galvanised pure Zn, galvanneal and Zn-Al alloy coatings on steel, Acta Materialia 51 (18) (2003) 5413-5424.

[58] H. Balzer, M. Hoehne, V. Sturm, R. Noll, Online coating thickness measurement and depth profiling of zinc coated sheet steel by laser-induced breakdown spectroscopy, Spectrochimica Acta Part B: Atomic Spectroscopy 60 (7-8) (2005) 1172-1178.

[59] J.-M. Mataigne, V. Vaché, M. Repoux, Surface chemistry and reactivity of skin-passed hot dip galvanized coating, Revue de Métallurgie-International Journal of Metallurgy 106 (1) (2009) 41-47.

[60] C. R. Tellier, Angular and surface roughness dependence of the electrical conductivity of thin metal films, Journal of Materials Science Letters 3 (5) (1984) 464-468. 


\title{
Supplementary Information: \\ Effect of surface roughness on the ultra short pulsed laser ablation fluence threshold of zinc and steel
}

\author{
H. Mustafa ${ }^{\mathrm{a}, *}$, M. Mezera ${ }^{\mathrm{a}}$, D.T.A. Matthews ${ }^{\mathrm{a}, \mathrm{b}, \mathrm{c}}$, G.R.B.E. Römer ${ }^{\mathrm{a}}$ \\ ${ }^{a}$ Chair of Laser Processing, Department of Mechanics of Solids, Surfaces 83 Systems (MS ${ }^{3}$ ), Faculty of \\ Engineering Technology, University of Twente, Enschede, the Netherlands \\ ${ }^{b}$ Chair of Skin Tribology, Department of Mechanics of Solids, Surfaces $\&$ Systems (MS ${ }^{3}$ ), Faculty of \\ Engineering Technology, University of Twente, Enschede, the Netherlands \\ ${ }^{c}$ Tata Steel Research and Development, IJmuiden, the Netherlands
}

\begin{abstract}
This document holds supplementary information related to the manuscript titled - Effect of surface roughness on the ultra short pulsed laser ablation fluence threshold of zinc and steel.
\end{abstract}

\section{Surface topography}

Figure S.1 shows the amplitude (height) density function (ADF) of the surfaces shown in Fig. 1 in Sec. 3.1. The skewness parameter of sample Zn2 indicates more scratches on the surface than sample Zn1. All the treated GI samples are leptokurtic while the as received sample (GI3) is platykurtic. The ADF of FS1 has similar trend as FS2 but at a smaller height scale. From the skewness parameters, it can be concluded that nearly all the samples exhibit depressions and scratches rather than peaks and spikes.

\footnotetext{
*Corresponding author at: P.O. Box 217, 7500 AE, Enschede, the Netherlands

Email address: h.mustafa@utwente.nl (H. Mustafa)
} 

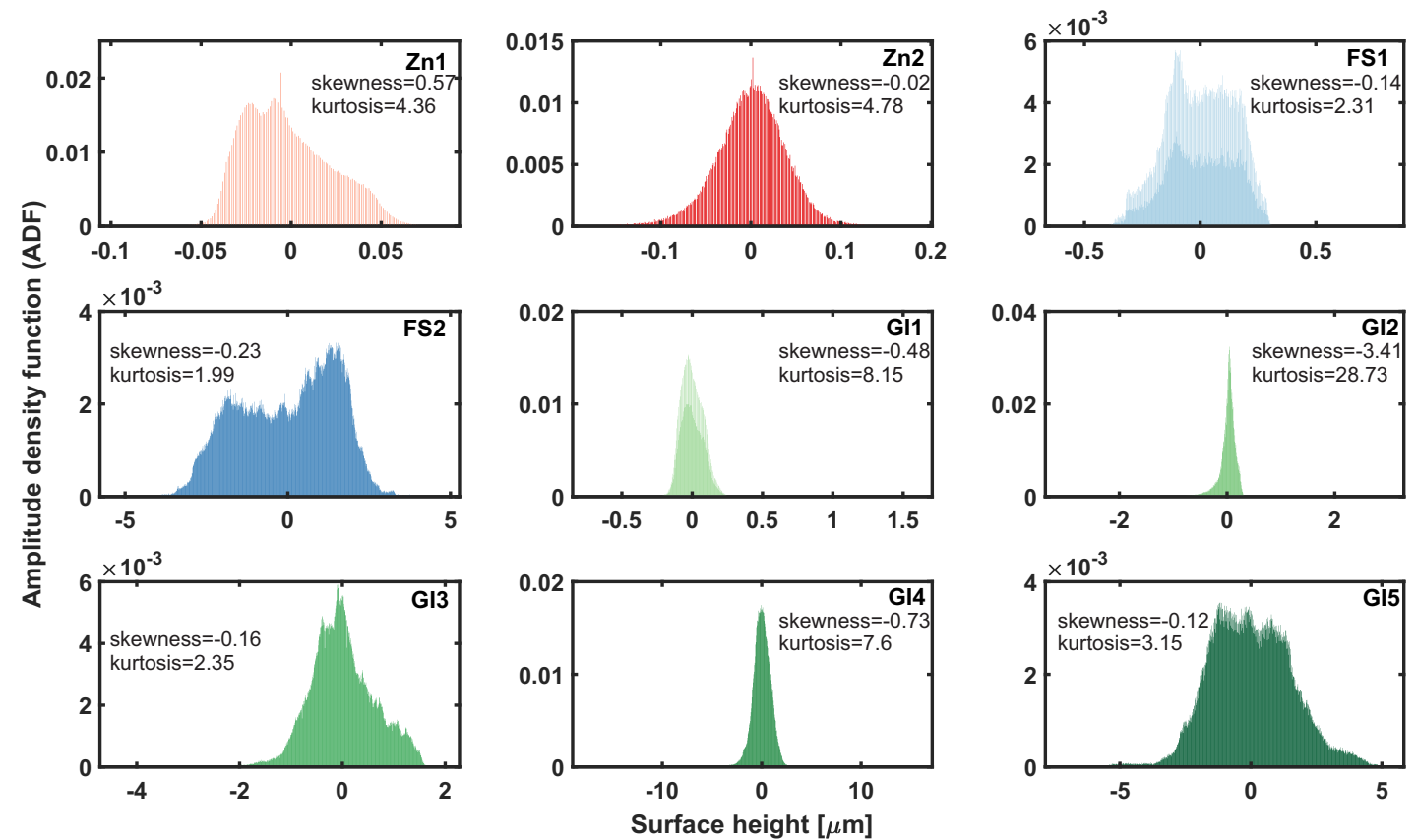

Supplemental Material, Figure S.1: Amplitude density function (ADF) of different material surfaces with corresponding statistical parameters skewness and kurtosis.
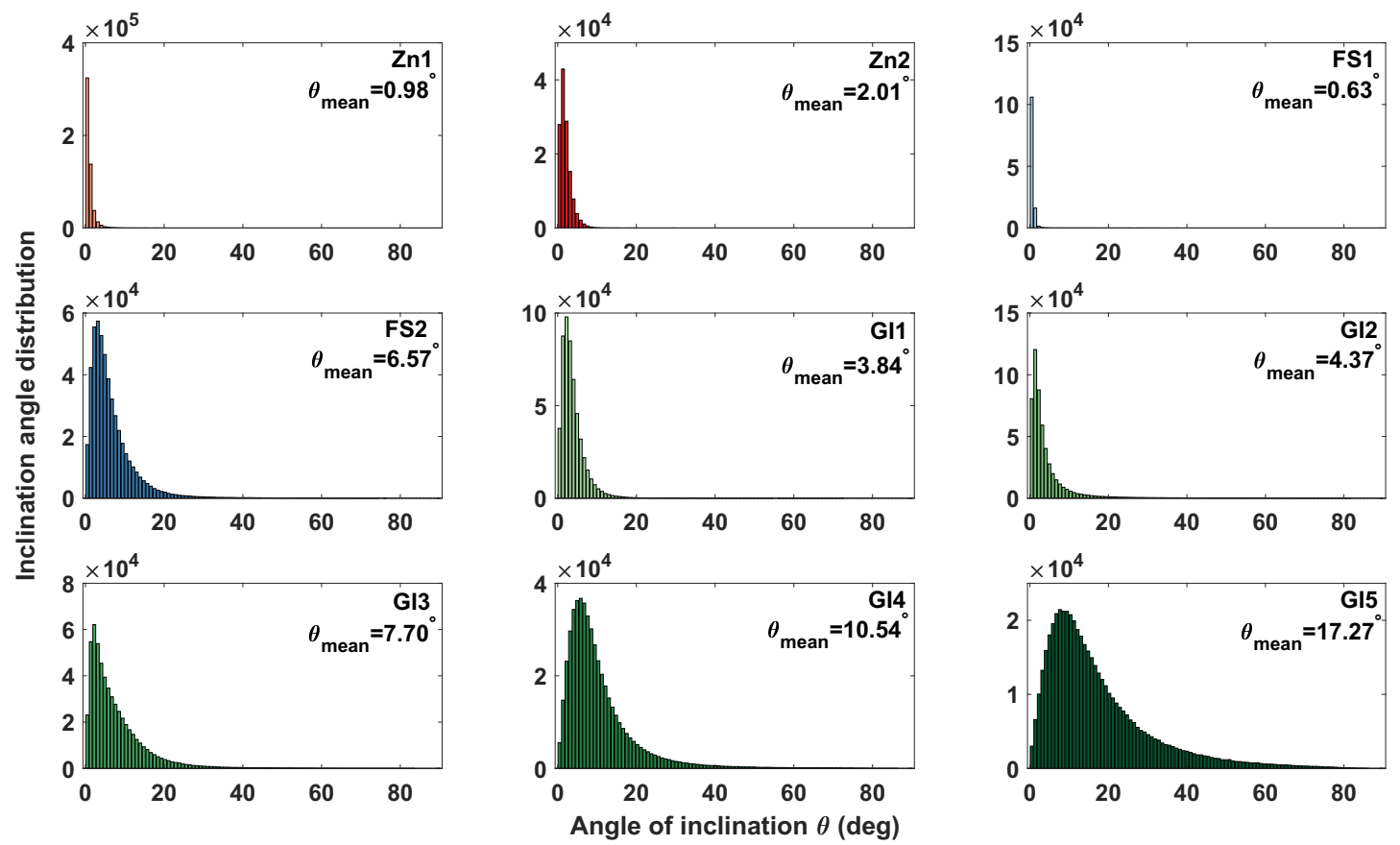

Supplemental Material, Figure S.2: Inclination angle distribution (IAD) function of different material surfaces. $\theta_{\text {mean }}$ denotes the average inclination angle of the distribution.

The inclination angle distribution (IAD) of the surfaces are shown in Fig. S.2. These 
local inclination angles determine the angle of incidence (AOI) of the incoming laser beam at each local surface point. The smoother the surface, the sharper the distribution in these graphs. Although samples FS2 and GI5 exhibit almost similar $R_{R M S}$, the IAD is wider for GI5 than FS2. This indicates that the transitions between peaks and valleys are more steep for sample FS2 than for sample GI5.

\section{Surface reflectivity}
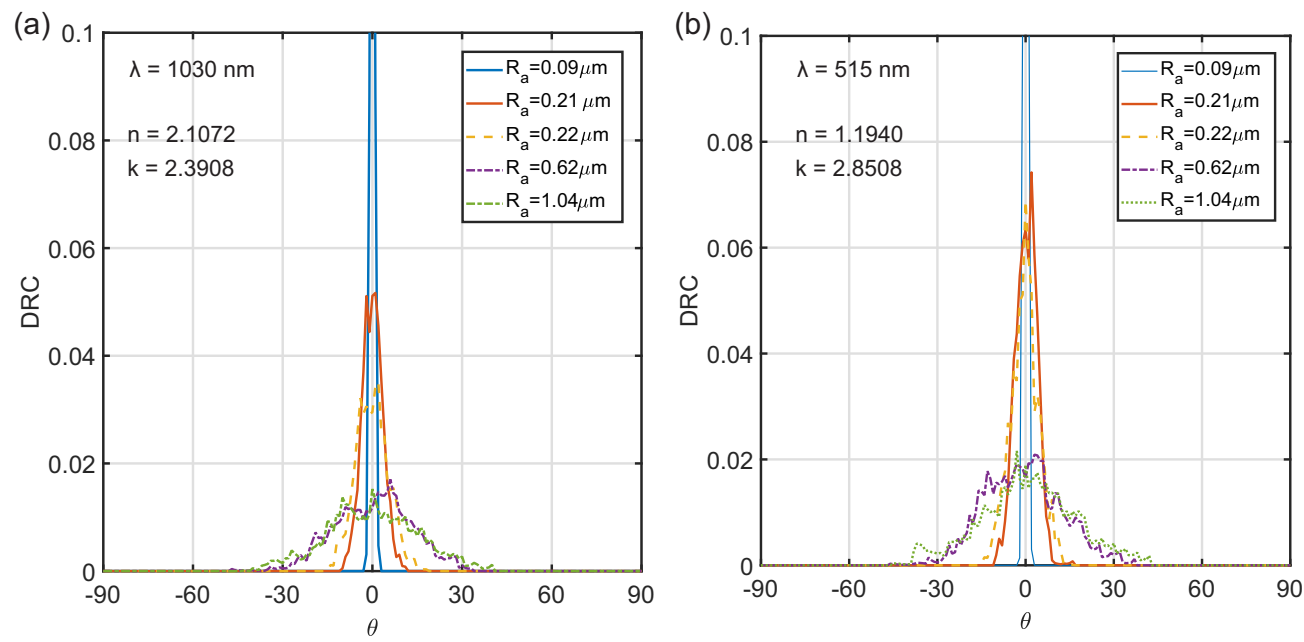

Supplemental Material, Figure S.3: Surface scattering plots for galvanized steel with different average surface roughness levels for light at normal incidence at a wavelength of (a) $1030 \mathrm{~nm}$ and (b) $515 \mathrm{~nm}$.

In geometric optics approximation, the angular distribution of scattered light is quantified using the directional reflection coefficient (DRC), which is the ratio of scattered light to incoming light at all scattering angles $\theta_{s}$ and is given by [1]

$$
\rho^{\prime \prime}\left(\cos \left(\theta_{s}\right)\right)=\frac{d \Phi_{s} / d \Omega_{s}}{d \Phi_{i} / d \Omega_{i}},
$$

where $\Phi$ and $\Omega$ are radiant powers and solid angles respectively, and the subscripts $i$ and $S$ denote the incident and the scattered ray respectively. In Fig. S.3, surface scattering plots of galvanized steel surfaces at different laser wavelengths are presented. The simulation results represent 40 surface realizations defined by 100000 points using 75000 first reflection points. In all the cases, the number of scattering events was singular, as expected for normal incidence light [1]. Reflection is dominantly specular for surfaces with a small RMS slope as the DRC follows a delta function for $\sigma / \tau_{x}=0.005$. This specular nature broadens with increasing surface roughness indicating more diffuse reflection. For $\sigma / \tau_{x}=0.03$ and 0.04, the scattered light distribution is bimodal in nature. The DRC for $\sigma / \tau_{x}=0.07$ and 0.09 resembles to the mean inclination angle shown in Fig. S.2. This means that the local 
angle of incidence plays the primary role in surface irregularity based absorption changes. However, geometric optics approximation is complimentary to exact wave-theoretical model for $\sigma \cos \left(\theta_{0}\right) / \lambda>0.17$ and $\sigma / \tau<2.0$ [2]. Although, the latter restriction is fulfilled by the surfaces studied in this work, the former restriction makes the first two curves in Fig. S.3(a) and only the first curve in Fig. S.3. (b) less valid for normal incidence $\left(\theta_{0}=0^{\circ}\right)$.
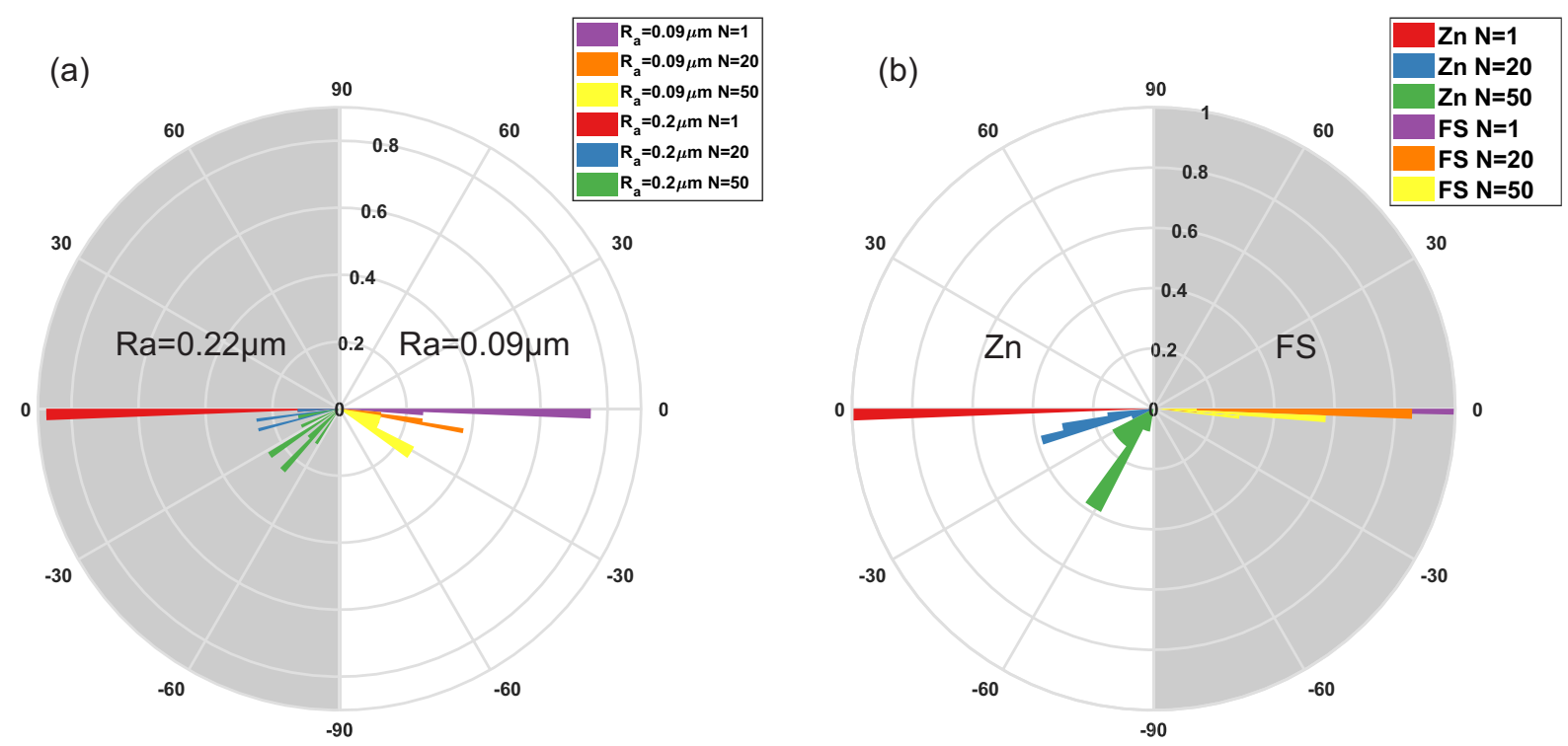

Supplemental Material, Figure S.4: Slope of the ablated craters - (a) galvanized steel at two different roughness, (b) zinc and forming steel polished surface.

With increasing fluence and/or number of pulses, dimensions of the ablated craters increase. This implies that the local angle of incidence is not normal for incoming light, rather it follows the slope of the ablated crater. Figure S.4 shows the polar histogram plot of the crater slopes at different number of pulses. The scatter for a given $N$ is a result of peak fluence difference. For a given material, increasing roughness does not affect the crater slope significantly. The difference observed for $N=50$ in Fig. S.4(a) is due to the reduction of coating thickness, which results in a shift from zinc to forming steel ablation. As can be observed from Fig. S.4(b), the increment in the crater slope with increasing $F_{0}$ and $N$ is insignificant for forming steel when compared to zinc. Therefore, the ablation rate of forming steel is less than of zinc. This results in a slower increase or saturated behavior in the development of crater slope in Fig. S.4(a). As the slope increases, a change in local angle of incidence results in higher absorption (see Fig. 2(d)) through diffuse reflection from laser induced structures on the crater wall as well as multiple-reflections [3, 4]. 


\section{Crater morphology}

For Zn, the surface modification is strongly affected by the initial surface roughness for $515 \mathrm{~nm}$ than $1030 \mathrm{~nm}$. While there is melt movement observable on the smoother sample (see Fig. S.5 (c)), on the rougher sample random bubble bursts within the laser irradiated zone can be observed (see Fig. S.5(d)).
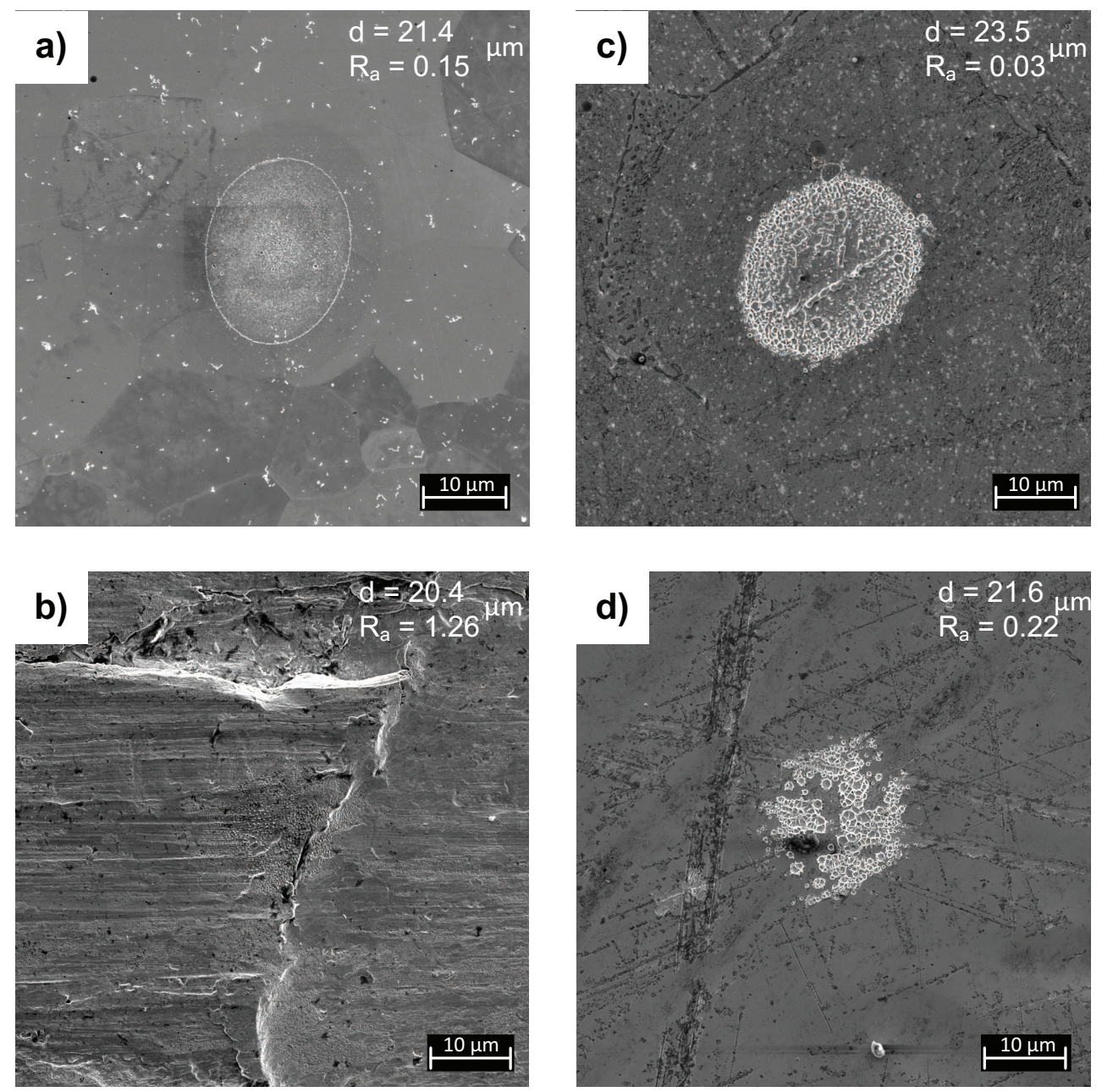

Supplemental Material, Figure S.5: SEM images (top view) of (a)-(b) forming steel and (c)-(d) zinc samples irradiated at a wavelength of $515 \mathrm{~nm}$ with $N=1$ and $F_{0}=2.1 \mathrm{~J} / \mathrm{cm}^{2}$. Diameter, $d$ of the modified surface and average surface roughness, $R_{a}$ of the unprocessed surface are derived from CLSM measurements. All images are in same scale. 

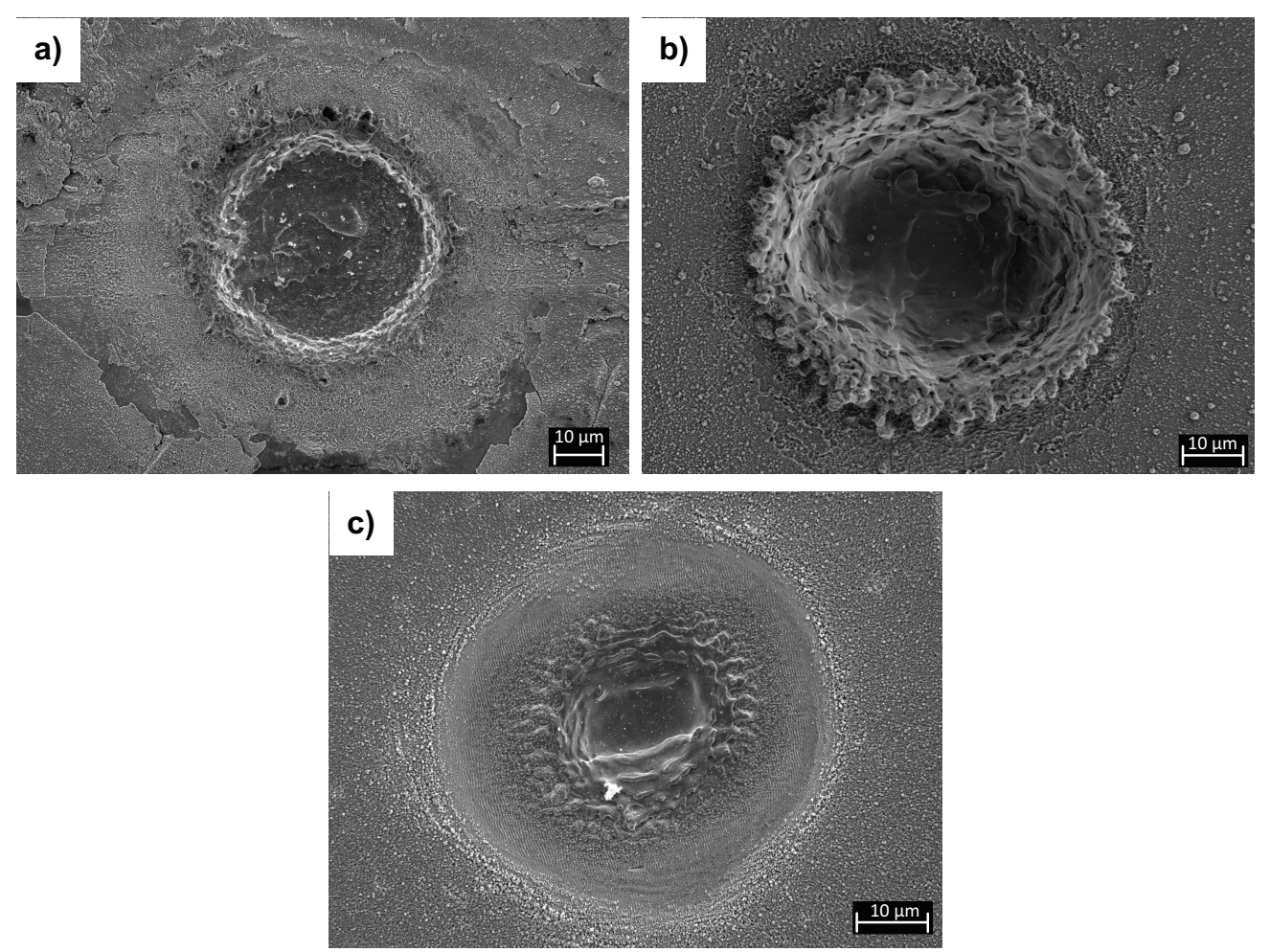

Supplemental Material, Figure S.6: SEM images (top view) of (a) GI, (b) Zn and (c) FS samples irradiated with $N=50$ and $F_{0}=40 \mathrm{~J} / \mathrm{cm}^{2}$.

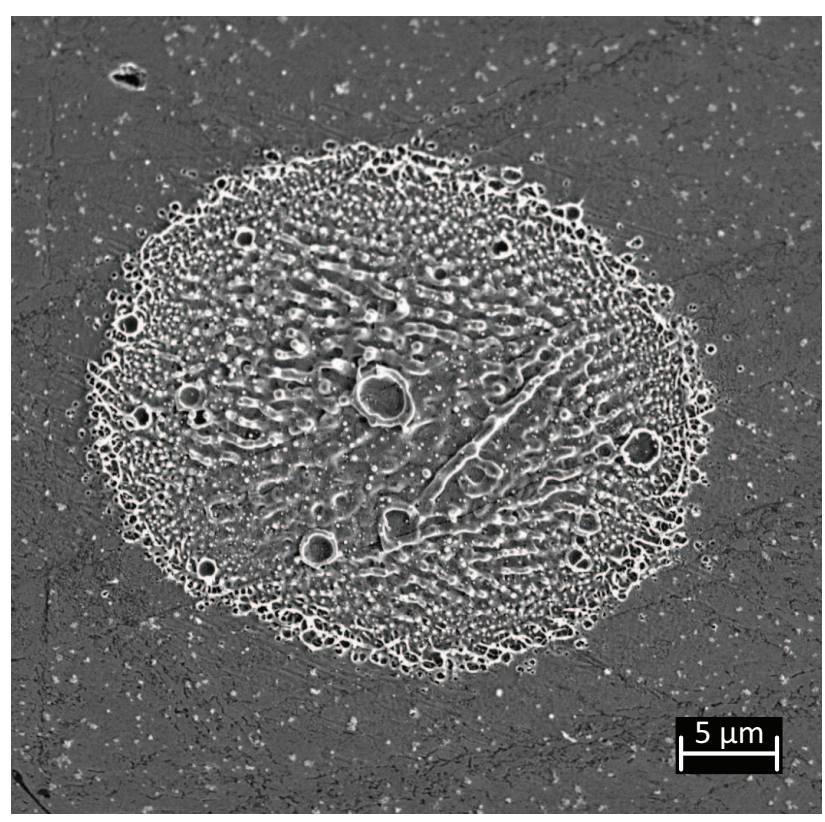

Supplemental Material, Figure S.7: SEM micrographs of LIPSS on laser processed Zn surface at a wavelength of $1030 \mathrm{~nm}$ and $N=1, F_{0}=2.75 \mathrm{~J} / \mathrm{cm}^{2}$. 
Table 1: Occurrence of LIPSS on different sample at different processing conditions. Tick and cross marks indicate presence and absence of LIPSS respectively.

\begin{tabular}{|c|c|c|c|c|c|c|c|c|c|c|c|c|c|}
\hline Fluence & \multicolumn{12}{|c|}{$F_{0}<10 \mathrm{~J} / \mathrm{cm}^{2}$} & \multirow{2}{*}{$\begin{array}{c}F_{0}>10 \mathrm{~J} / \mathrm{cm}^{2} \\
1030 \mathrm{~nm}+515 \mathrm{~nm}\end{array}$} \\
\hline Wavelength & & & 103 & $\mathrm{~nm}$ & & & & & & $\mathrm{~nm}$ & & & \\
\hline Number of pulses & 1 & 5 & 10 & 15 & 20 & 50 & 1 & 5 & 10 & 15 & 20 & 50 & $1-50$ \\
\hline FS1, FS2 & $x$ & $\checkmark$ & $\checkmark$ & $\checkmark$ & $\checkmark$ & $\checkmark$ & $x$ & $\checkmark$ & $\checkmark$ & $\checkmark$ & $\checkmark$ & $\checkmark$ & $x$ \\
\hline Zn1 & $\checkmark$ & $x$ & $x$ & $x$ & $x$ & $x$ & $x$ & $x$ & $x$ & $x$ & $x$ & $x$ & $x$ \\
\hline Zn2, GI1-5 & $x$ & $x$ & $x$ & $x$ & $x$ & $x$ & $x$ & $x$ & $x$ & $x$ & $x$ & $x$ & $x$ \\
\hline
\end{tabular}

(a)

GI - 1030 nm

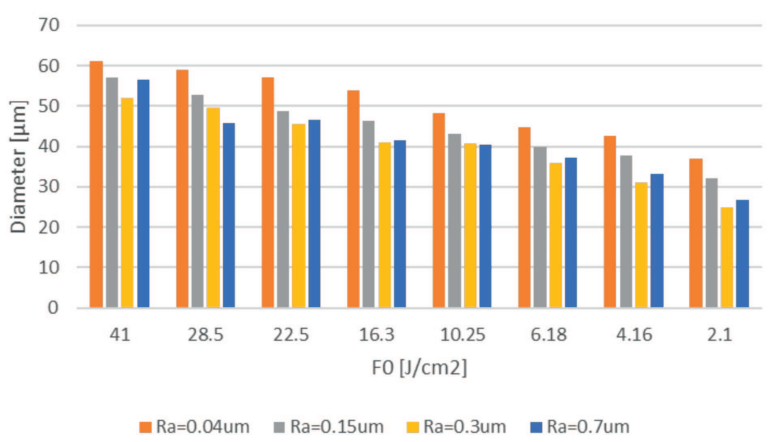

(b)

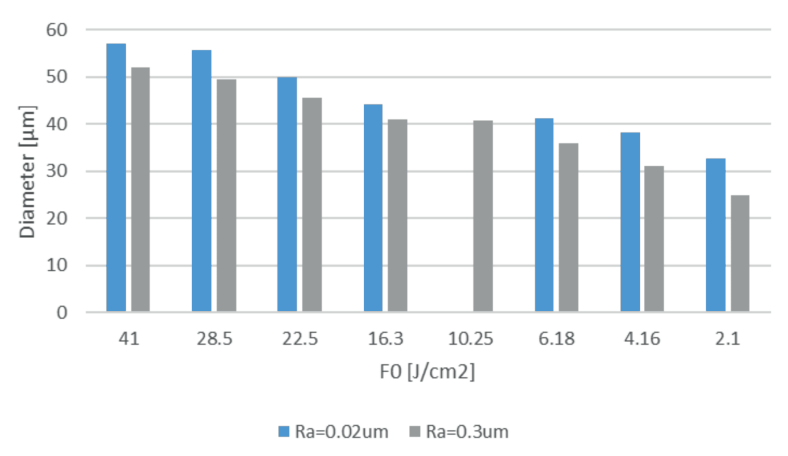

Supplemental Material, Figure S.8: Evolution of crater diameter with laser peak fluence for different initial surface roughness at a wavelength of $1030 \mathrm{~nm}$ in (a) galvanized steel and (b) bulk zinc. 


\section{Ablation threshold}
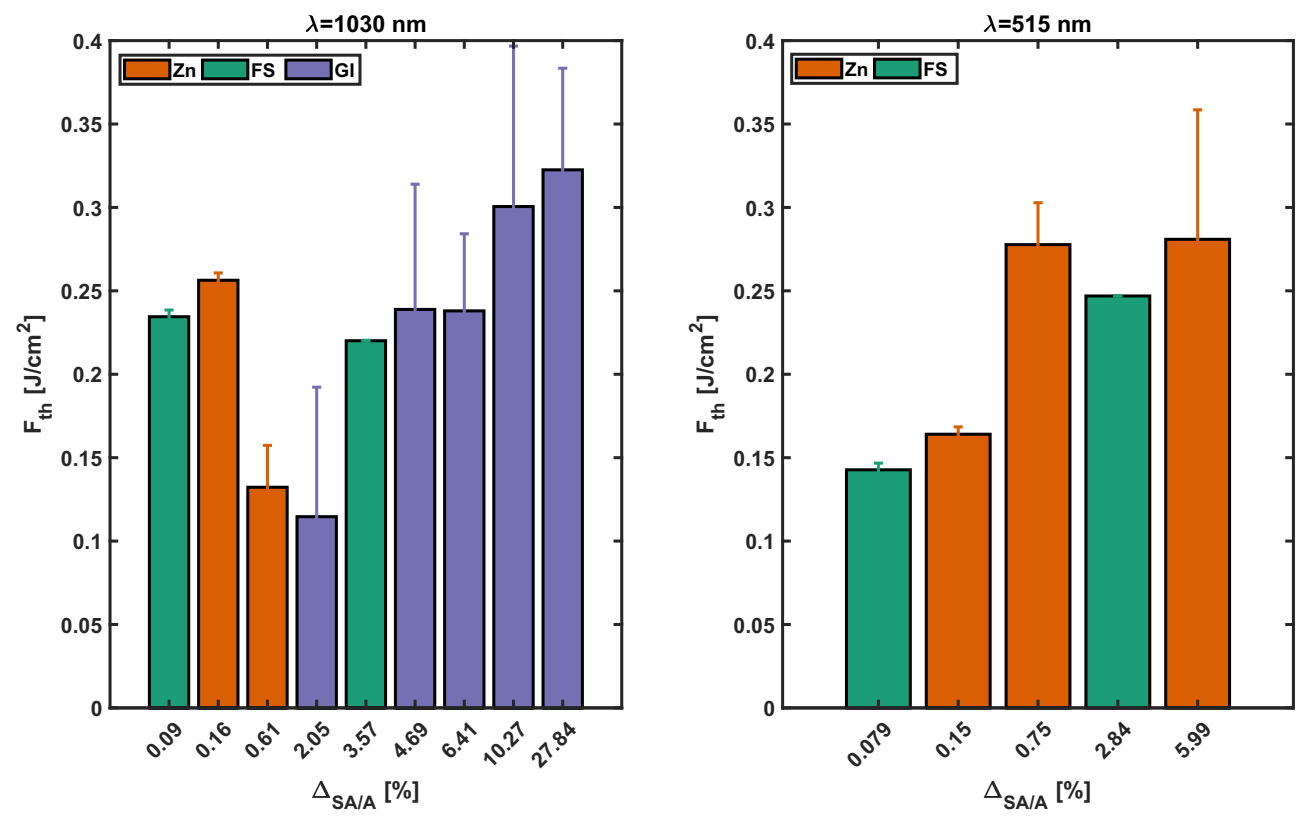

Supplemental Material, Figure S.9: Threshold fluence $F_{t h}(N)$ for $N=10$ as a function of increase in surface area $\Delta_{S A / A}$.

The effect of initial surface roughness and resulting incubation is also reflected in multipulse irradiation as shown in Fig. S.9. Here, a similar trend as for $N=1$ in Fig. 6 is observed for $N=10$. However, the difference in threshold fluence requirement with increasing $\Delta_{S A / A}$ becomes smaller for $N>1$. This is because of intensity redistribution through multiple and diffuse reflection, as a well defined crater is formed [3, 4].

\section{Heat accumulation model}

The temperature distribution for a spatial Gaussian shaped stationary beam after $N$ pulses is given by [5],

$$
T(x, y, z, t)=\sum_{n=0}^{N} T^{s . p .}\left(x, y, z, t+n \Delta t_{p-p}\right),
$$

where $t_{p-p}$ is the time between laser pulses and $T^{s . p .}$ at the surface $(z=0)$ is given by [5],

$$
T^{s . p .}(x, y, z=0, t)=\frac{2 E_{\text {res }}}{\pi \rho C_{p} \sqrt{\pi \kappa t}\left(8 \kappa t+\omega_{0}^{2}\right)} e^{\frac{\left(x-x_{c}\right)^{2}+\left(y-y_{c}\right)^{2}}{4 \kappa t}\left(\frac{\omega_{0}^{2}}{8 \kappa t+\omega_{0}^{2}}-1\right)},
$$

where $\left(x_{c}, y_{c}\right)$ are center coordinates of the laser spot, $\rho, C_{p}, \kappa$ and $\omega_{0}$ are density, specific heat capacity, thermal diffusivity of the material and beam radius respectively. The residual 
energy $E_{\text {res }}$ is the product of incident pulse energy $E_{p}$ and residual rate of single pulse energy $\eta_{\text {res }}$ given by [6],

$$
\eta_{\text {res }}=\frac{A E_{p}-\rho \Delta V L_{v a p}}{E_{p}} \times 100 \%,
$$

where $L_{v a p}$ is the latent heat of vaporization and $\Delta V$ is the volume ablation rate per pulse. From the confocal data, $\Delta V$ is calculated as [7, 8]

$$
\Delta V=\frac{1}{4} \pi \omega_{t o t}^{2} \delta_{e}\left[\ln \left(\frac{E_{p} /\left(\pi \omega_{t o t}^{2}\right)}{F_{t h}}\right)\right]^{2},
$$

where $\omega_{t o t}=\omega_{0}+(S A / A-1) \cdot \omega_{0}$ and $\delta_{e}$ is energy penetration depth.

\section{References}

[1] D. Bergström, J. Powell, A. F. H. Kaplan, A ray-tracing analysis of the absorption of light by smooth and rough metal surfaces, Journal of Applied Physics 101 (11) (2007) 113504.

[2] K. Tang, R. A. Dimenna, R. O. Buckius, Regions of validity of the geometric optics approximation for angular scattering from very rough surfaces, International Journal of Heat and Mass Transfer 40 (1) (1996) 49-59.

[3] A. Vorobyev, C. Guo, Reflection of femtosecond laser light in multipulse ablation of metals, Journal of Applied Physics 110 (4) (2011) 043102.

[4] A. Y. Vorobyev, C. Guo, Thermal response and optical absorptance of metals under femtosecond laser irradiation, Natural Science 3 (06) (2011) 488-495.

[5] F. Bauer, A. Michalowski, T. Kiedrowski, S. Nolte, Heat accumulation in ultra-short pulsed scanning laser ablation of metals, Optics express 23 (2) (2015) 1035-1043.

[6] B. Wu, P. Liu, X. Wang, F. Zhang, L. Deng, J. Duan, X. Zeng, Effect of laser absorption on picosecond laser ablation of $\mathrm{Cr} 12 \mathrm{MoV}$ mold steel, $9 \mathrm{Cr} 18$ stainless steel and H13A cemented carbide, Optics \& Laser Technology 101 (2018) 11-20.

[7] B. Neuenschwander, G. Bucher, G. Hennig, C. Nussbaum, B. Joss, M. Muralt, S. Zehnder, U. W. Hunziker, P. Schuetz, Processing of dielectric materials and metals with ps laser pulses, in: Proceedings of the 29th International Congress on Applications of Lasers \& Electro-Optics (ICALEO), Anaheim, California, 2010.

[8] G. Račiukaitis, M. Brikas, P. Gecys, B. Voisiat, M. Gedvilas, et al., Use of high repetition rate and high power lasers in microfabrication: How to keep the efficiency high?, JLMN Journal of Laser Micro/Nanoengineering 4 (3) (2009) 186-191. 